\title{
Identification and Characterization of the Grape WRKY Family
}

\author{
Ying Zhang and Jian can Feng \\ Forestry Department, Agricultural University of Henan Province, Zhengzhou, Henan 450002, China \\ Correspondence should be addressed to Jian can Feng; jcfeng@henau.edu.cn
}

Received 15 November 2013; Revised 16 January 2014; Accepted 21 January 2014; Published 27 April 2014

Academic Editor: Anton M. Jetten

Copyright (c) 2014 Y. Zhang and J. C. Feng. This is an open access article distributed under the Creative Commons Attribution License, which permits unrestricted use, distribution, and reproduction in any medium, provided the original work is properly cited.

\begin{abstract}
WRKY transcription factors have functions in plant growth and development and in response to biotic and abiotic stresses. Many studies have focused on functional identification of WRKY transcription factors, but little is known about the molecular phylogeny or global expression patterns of the complete WRKY family. In this study, we identified 80 WRKY proteins encoded in the grape genome. Based on the structural features of these proteins, the grape WRKY genes were classified into three groups (groups 1-3). Analysis of WRKY genes expression profiles indicated that $28 W R K Y$ genes were differentially expressed in response to biotic stress caused by grape whiterot and/or salicylic acid (SA). In that 16 WRKY genes upregulated both by whiterot pathogenic bacteria and SA. The results indicated that 16 WRKY proteins participated in SA-dependent defense signal pathway. This study provides a basis for cloning genes with specific functions from grape.
\end{abstract}

\section{Introduction}

Various defense mechanisms have evolved in plants to combat microbial infection. Transcription factors are central to this process, and transcription factor families have expanded and evolved in plants to coordinate gene expression. Expression of a large number of defense-related plant genes is regulated at the transcriptional level in response to pathogen infection [1]. Timely transcriptional regulation of defenserelated genes is crucial for effective responses to pathogens [2]. Proteins of the WRKY family are the most important transcription factors for the regulation of plant defense response pathways [3-5]. The WRKY name is derived from the conserved DNA binding domain sequence WRKYGQK; the conservative domain is approximately 60 residues, followed by a Cys2His2 or Cys2HisCys zinc-binding motif $[6,7]$, and WRKY proteins were divided into Group I-III based on the number of WRKY domains and the structure of zinc fingers $[8,9]$. The Group II WRKY proteins are classified into $\mathrm{a}, \mathrm{b}, \mathrm{c}, \mathrm{d}$, and e subgroups based on their zinc finger motifs [5].

In higher plants, the WRKY gene family members play a variety of roles. Accumulating evidence indicates that WRKY transcription factors are involved in responses to biotic stresses as well as in plant development $[7,10,11]$. Salicylic acid (SA) is an response endogenous phytohormone and an important signal substances in the deployment of systemic acquired resistance (SAR) [12]. SAR is characterized by an increase in endogenous salicylic acid (SA) and enhanced resistance to a broad spectrum of virulent pathogens. SA is necessary for SAR, and a series of studies demonstrated that SA triggers host defence mechanisms against pathogen infections $[13,14]$. Many WRKY genes are key factors controlling plant response to disease resistance especially pathogen infections that can trigger SA-dependent defense signaling. In Arabidopsis, WRKY70 was identified as an important node of SA signaling during plant defense responses [15]. In addition, treatment of Arabidopsis with a bacterial pathogen or salicylic acid (SA) resulted in differential expression of WRKY genes $[15,16]$. This pattern of expression was also observed in other plant species [10, 17-19].

Cultivated grapevines are susceptible to many pathogens including phytoplasmas, viruses, bacteria, and fungi [22]. Among these, grape white rot (Coniothyrium diplodiella) is the most important agriculturally because it causes extensive losses in quantity and quality of harvested berries. As a consequence, table and wine grape cultivation requires extensive use of phytochemicals. In China, grape white rot is the main fungal disease of grapes causing heavy losses in 
grape production [23]. Vitis vinifera L. ("European" grape) is the most economically important and widespread species of Vitis spp. producing more than $90 \%$ of world's production of table, wine, and raisin grapes. More than $80 \%$ of cultivated grape varieties are derived from this species. All $V$. vinifera $\mathrm{L}$. varieties are susceptible to grape white rot [24]. The disease is found in most grape-growing regions of the world [25] and results in poor berry quality and weakened vines in warm and humid climates. Multiple research strategies are currently being pursued so that wine growers may produce healthy fruits right up to maturity with minimum use of chemical treatments.

The complete $V$. vinifera PN40024 genomic sequence $[26,27]$ has been determined. The $V$. vinifera genome was the first fruit tree species genome to be sequenced, making $V$. vinifera an ideal model system for fruit trees [26]. The release of the latest $V$. vinifera genome sequence, $12 \mathrm{X}$ (http:// www.genoscope.cns.fr/externe/GenomeBrowser/Vitis/), and the many WRKY gene and protein sequences from other Vitis spp. present in the NCBI (http://www.ncbi.nlm.nih .gov/) and PlantTFDB (http://planttfdb.cbi.pku.edu.cn/index .php? sp=Vvi) databases provided an opportunity to analyze and further understand the grape $W R K Y$ gene family. In this study, we identified a comprehensive and nonredundant set of eighty WRKY genes in the grape genome. Phylogenetic and motif analysis and characterization of WRKY expression induced by pathogens and salicylic acid (SA) were also performed to lay a solid foundation for further comparative genomics studies.

\section{Materials and Methods}

2.1. Characterization of Putative WRKY Proteins in Grape. The latest $12 \mathrm{X} V$. vinifera genomic and protein sequences were downloaded from the NCBI database. The procedure used to survey grape WRKY proteins was similar to identification methods described for other species. The hidden Markov model (HMM) profile for the WRKY domain from the Pfam database was used as a query to survey all potential proteins. The Pfam database was then used to decide if the candidate proteins contained features typical of WRKY proteins. Identical and defective sequences were eliminated using manual inspection in the MEGA ver4.0 software. Nonoverlapping WRKY protein sequences were used for further analysis.

\subsection{Phylogenetic Analysis Based on Conserved WRKY} Domains. WRKY genes of was retrieved by TBlastN software from the publicly available information in the database (http://www.ncbi.nlm.nih.gov, http://www.genoscope.cns .fr/externe/GenomeBrowser/Vitis/). Conserved WRKY domains of the VvWRKY proteins were identified by manual inspection using the Pfam software and used to generate a multiple sequence alignment of the WRKY domains. Phylogenetic trees based on 58 representative domains from poplar, Arabidopsis, rice, and grape were constructed using Clustal X ver1.83 and Mega ver4.0 [28] by the neighborjoining $(\mathrm{NJ})$ method to produce improved classifications of the different clades. Bootstrap values were calculated from 1,000 iterations.

2.3. Analysis of Conserved Motif Distribution and Structure and Gene Duplication in Grape. To assess the structural divergence of $V v W R K Y$ genes, conserved motifs in the encoded proteins were identified using the multiple expectation maximization for motif elicitation (MEME) online software (http://meme.sdsc.edu/meme/intro.html). Parameters employed in the analysis were as follows: maximum number of motifs, 20; minimum motif width, 6; and maximum motif width, 50. The online software 2ZIP (http://2zip.molgen.mpg.de/index.html) was used to predict the conserved Leu zipper motif. HARF, LXXLL, and LXLXLX motifs were identified manually.

2.4. Expression Analysis. Chinese wild grape V. vinifera "Pinor" leaves (numbered by the National Repository for Grapevine (Zhengzhou grape germplasm repository)) were used for all experiments. When shoots of vines were 25$35 \mathrm{~cm}$ in length, the third through fifth fully expanded young leaves beneath the apex were selected for biotic and abiotic treatments. The plants were subjected to abiotic stress and SA treatments according to Ramamoorthy et al. [29]. For SA treatment, plants were sprayed with a $0.1 \mathrm{mM}$ SA solution. Treatment with deionized water served as a control and was repeated three times on three independent plants. Leaves were collected $0,9,12,24$, and $48 \mathrm{~h}$ after treatment and immediately frozen in liquid nitrogen for further study.

For pathogen treatment, leaves were inoculated with Coniothyrium diplodiella mycelium gelose discs from a 3day-old culture at six sites and placed on PDA medium at $28^{\circ} \mathrm{C}$. Leaves sprayed with PDA medium were used as negative control. Leaves were collected $0,9,12,24$, and $48 \mathrm{~h}$ after inoculation (hpi). Treatment with deionized water was performed as a control. After harvest, the materials were immediately frozen in liquid nitrogen and stored at $-80^{\circ} \mathrm{C}$ for further analysis.

Grape total RNA was extracted as previously described [30]. Grapevine total RNA was extracted from $V$. vinifera "Pinor" leaves using an improved SDS/phenol method [31] at $0,9,12,24$, and $48 \mathrm{~h}$ after infection with Coniothyrium diplodiella. The following primers were used for RTPCR amplification: 5'-GCGGGCAAGAGATACCTCAA$3^{\prime}$ and $5^{\prime}$-TCAATCTGTCTAGGAAAGGAAG-3' for EFl $\gamma$ (AF176496). Three independent PCR reactions were carried out for each gene and similar results were obtained. Amplification products were quantified using a Roche 480 II realtime PCR instrument.

\section{Results}

3.1. Identification of WRKY Proteins from Grape. To identify WRKY proteins encoded in the grape genome, publicly available genome sequences were searched using the BlastP software based on an HMM (PF03106). A total of 80 putative grape WRKY protein sequences were initially identified. 
Group I NT

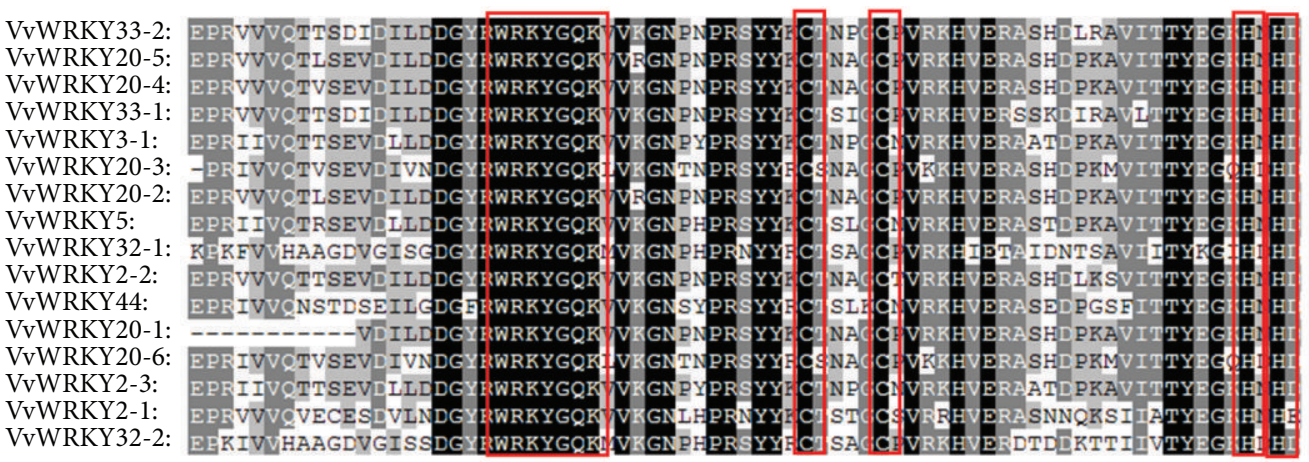

Group I CT:

VvWRKY3-1: VvWRKY5:

VvWRKY20-1:

VvWRKY2-2:

VvWRKY20-2:

VvWRKY2:

VvWRKY20-5:

VvWRKY32:

VvWRKY20-3:

VvWRKY44:

VvWRKY33-1:

VvWRKY2-1:

VvWRKY20-6:

VvWRKY33-2:

VvWRKY20-4

VvWRKY2-3:

VvWRKY5:

VvWRKY32-2:
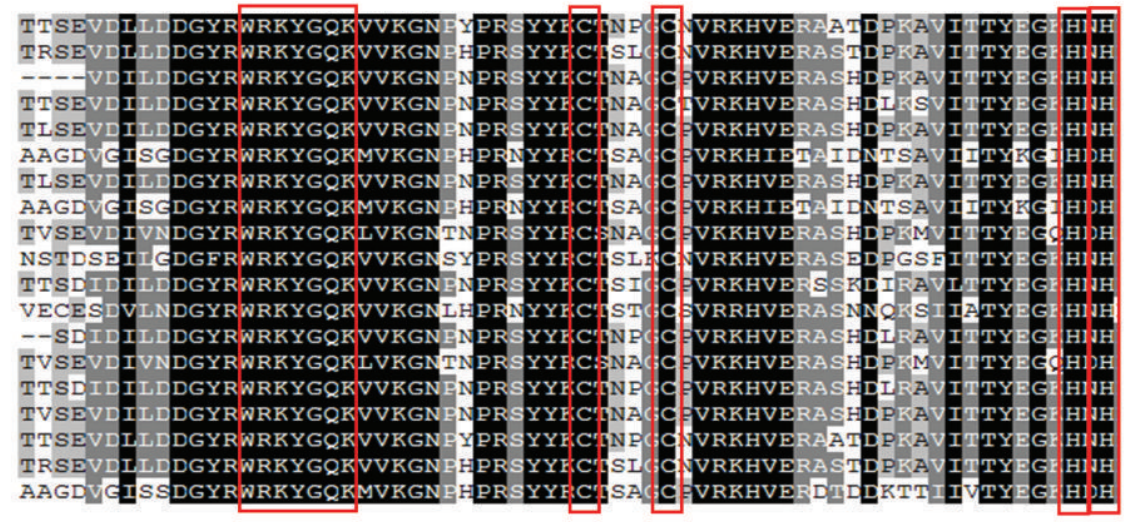

Group IIa

VvWRKY18:

VvWRKY3-2

VvWRKY40-2

VvWRKY40-1

VvWRKY4

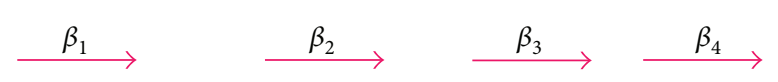

Group IIb

VvWRKY6-2:

VvWRKY42:

VvWRKY6-1:

VvWRKY9:

VvWRKY72-2:

VvWRKY72-3:

VvWRKY72-1:

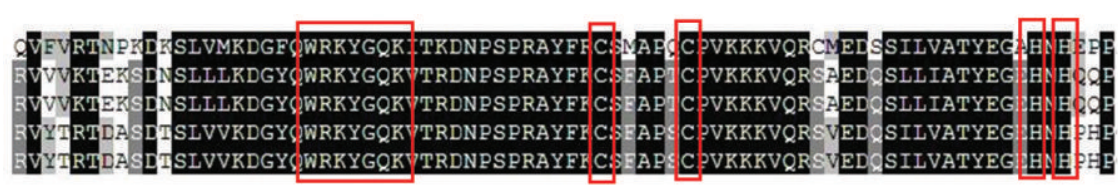

Group IIc:

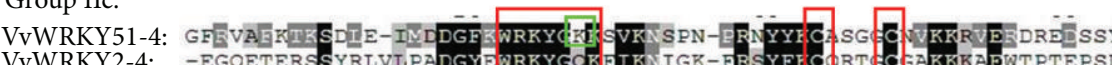

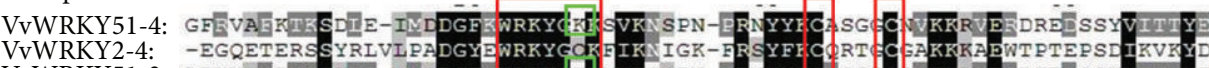

VVWRKY51-3: ARVVAERTKSEID-VNDDGFKWRKY

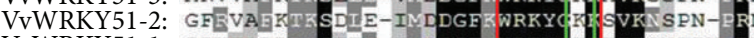

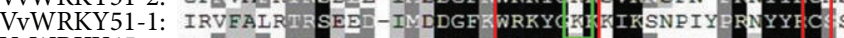

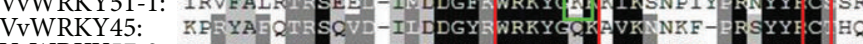

VWWKY57-2:

VVWRKY57-2: KPRYAEQTISSVI-IIDDGYRWRKYGCRAVKNNRF- ER

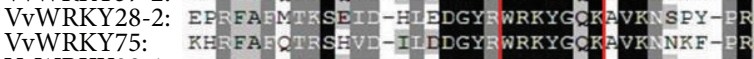

VVWRKY28-1: EPRFAEM

VyWRKY1-2:

VvWRKY57:

VvWRKY50:

VvWRKY48:

VvWRKY24:

VvWRKY23:

VvWRKY49:

VvWRKY12:

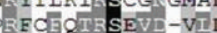

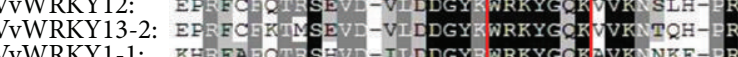

VyWRKY12-2.

VvWRKY13:

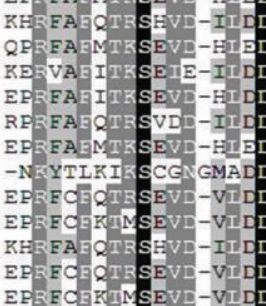

KNRKYGDK

KN $\mathrm{TH}-\mathrm{PH}-\mathrm{P}$

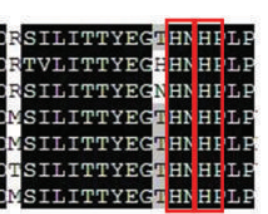

Figure 1: Continued. 


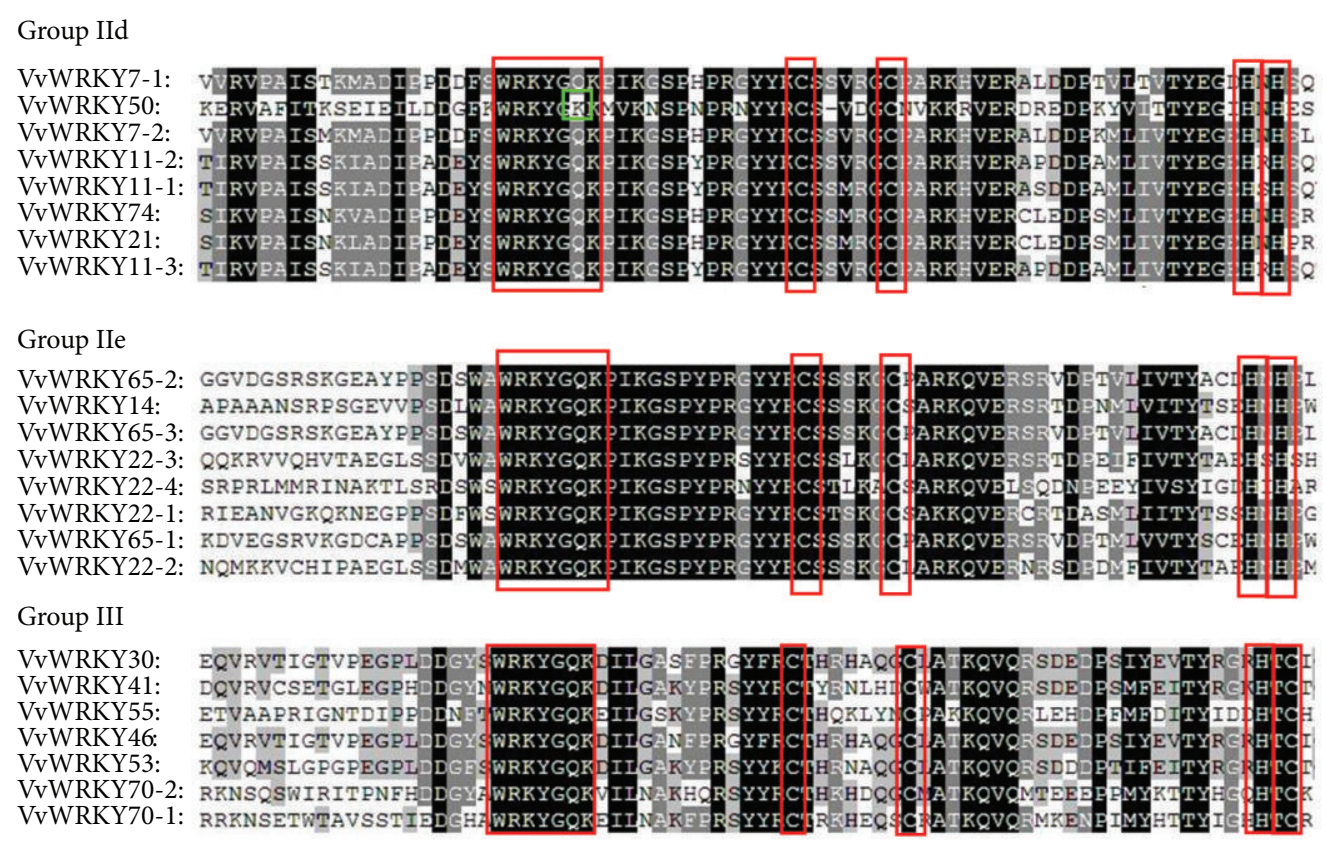

(a)
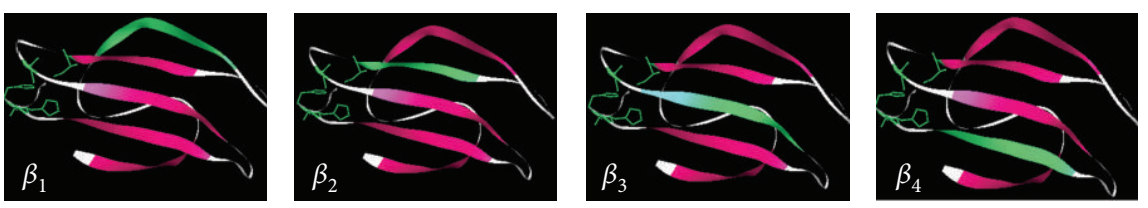

(b)

FIGURE 1: The WRKY conservative domain. (a) Comparison of WRKY domain sequences from VvWRKY proteins. Sequences encoding the peptide stretch WRKYGQK were found by the BLAST programs tblastn and blastp programs in genomic and EST databases. Gaps (dots) have been inserted for optimal alignment. Residues that are highly conserved within each of the major groups are in black and potential zinc ligands are highlighted in red boxes, and the different amino acid residues are highlighted in green boxes. (b) The four $\beta$-strands are shown in red. I CT and I NT denote the N- and C-terminal WRKY domains from Group I WRKY proteins. As in (b), the $\beta_{1}, \beta_{2}, \beta_{3}$, and $\beta_{4}$ motifs are shown in green and the $\mathrm{Zn}$ finger is indicated by a green line.

With the exception of VvWRKY1, VvWRKY2, VpWRKY1, and VPWRKY2, none of the proteins were described previously. Based on manual inspection using the MEGA ver4.0 software, seven sequences were discarded due to redundancy or insufficient conservation of WRKY sequences. The remaining WRKY proteins were reviewed using the Pfam program to confirm that all candidates contained the conserved WRKY motif. Properties of the proteins including numbers of amino acids, molecular weights, and isoelectric points (PI) are listed in Table 1. The average VvWRKY sequence length was 382 amino acids and lengths ranged from 151 (VvWRKY1-1) to 798 (VvWRKY9) residues, while the isoelectric points (PI) ranged from 4.7 (VvWRKY22-4) to 9.84 (VvWRKY21).

The WRKY domain is approximately 60 amino acid residues in length and is considered to be a crucial element for interaction with the $\mathrm{W}$-box (C/T)TGAC(T/C) to activate many defense-related genes. In our study, $80 \mathrm{WRKY}$ domains contained highly conserved WRKYGQK sequences, while the other WRKY domains had one amino acid mismatch in the conserved WRKY sequence (Figure 1). In VvWRKY51-2,
51-3, and 51-3, the WRKY domain sequence was WRKYGKK. As described by Eulgem et al. [7], the metal-chelating zinc finger motif $\left(\mathrm{C}-\mathrm{X}_{4-5}-\mathrm{X}_{22-23}-\mathrm{H}-\mathrm{X}-\mathrm{H}\right.$ or $\mathrm{C}-\mathrm{X}_{5-8}-\mathrm{C}-\mathrm{X}_{25-28}-\mathrm{H}-\mathrm{X}_{1-2}-$ C) is another important characteristic of WRKY proteins. Zinc-finger-like motifs were identified in all of the grape WRKY proteins.

\subsection{Phylogenetic Analysis and Classification of WRKY Groups} in Grape. A phylogenetic tree was constructed based on the conserved WRKY domains to examine the phylogenetic relationships among all 80 members. VvWRKY domains of the Group I include two domains (the C-terminal and $\mathrm{N}$ terminal domain). A multiple sequence alignment of the 80 WRKY domains was performed (Figure 1). Three major groups were identified as described by $\mathrm{Wu}$ et al. [20]. In addition, several subgroups were revealed by the phylogenetic analysis.

The positions of the C- and N-terminal WRKY domains in the WRKY proteins were relatively consistent. Group I contained 19 WRKY proteins all of which contained two WRKY domains. Three Group I-CTWD (C-terminal 
TABLE 1: Members of the grape WRKY superfamily of transcription factors.

\begin{tabular}{|c|c|c|c|c|c|c|c|c|c|}
\hline \multirow{2}{*}{ Name } & \multirow{2}{*}{ Protein $^{\mathrm{a}}$} & \multirow{2}{*}{ Chr } & \multicolumn{3}{|c|}{ Deduced polypeptide } & \multirow{2}{*}{ Organism } & \multirow{2}{*}{ Group } & \multicolumn{2}{|c|}{ Domain } \\
\hline & & & Length (aa) & PI & MW (kDa) & & & Family name & Pattern \\
\hline VvWRKY 1-1 & GI:50953501 & * & 151 & 9.61 & 17.7 & Vitis vinifera & IIc & $1-1$ & $\mathrm{C}-\mathrm{X}_{4}-\mathrm{C}-\mathrm{X}_{23}-\mathrm{HXH}$ \\
\hline VvWRKY 1-2 & GI:50953502 & * & 297 & 9.67 & 17.7 & Vitis vinifera & IIc & $1-2$ & $\mathrm{C}-\mathrm{X}_{4}-\mathrm{C}-\mathrm{X}_{23}-\mathrm{HXH}$ \\
\hline VvWRKY 2-1 & GI:359476618 & 4 & 700 & 6.52 & 75.9 & Vitis vinifera & $\begin{array}{l}\text { I-NTWD } \\
\text { I-CTWD }\end{array}$ & $\begin{array}{l}2-1 \mathrm{~N} \\
2-1 \mathrm{C}\end{array}$ & $\begin{array}{l}\mathrm{C}-\mathrm{X}_{4}-\mathrm{C}-\mathrm{X}_{22}-\mathrm{HXH} \\
\mathrm{C}-\mathrm{X}_{4}-\mathrm{C}-\mathrm{X}_{23}-\mathrm{HXH}\end{array}$ \\
\hline VvWRKY 2-2 & GI:225463536 & 19 & 734 & 5.7 & 80.3 & Vitis vinifera & $\begin{array}{l}\text { I-NTWD } \\
\text { I-CTWD }\end{array}$ & $\begin{array}{l}2-1 \mathrm{~N} \\
2-2 \mathrm{C}\end{array}$ & $\begin{array}{l}\mathrm{C}-\mathrm{X}_{4}-\mathrm{C}-\mathrm{X}_{22}-\mathrm{HXH} \\
\mathrm{C}-\mathrm{X}_{4}-\mathrm{C}-\mathrm{X}_{23}-\mathrm{HXH}\end{array}$ \\
\hline VvWRKY 2-3 & GI:225469228 & $*$ & 536 & 7.21 & 58.44 & Vitis vinifera & $\begin{array}{l}\text { I-NTWD } \\
\text { I-CTWD }\end{array}$ & $\begin{array}{l}2-3 N \\
2-3 C\end{array}$ & $\begin{array}{l}\mathrm{C}-\mathrm{X}_{4}-\mathrm{C}-\mathrm{X}_{22}-\mathrm{HXH} \\
\mathrm{C}-\mathrm{X}_{4}-\mathrm{C}-\mathrm{X}_{23}-\mathrm{HXH}\end{array}$ \\
\hline VvWRKY 2-4 & GI:359478811 & 6 & 336 & 6.31 & 21.7 & Vitis vinifera & IIc & $2-4$ & $\mathrm{C}-\mathrm{X}_{4}-\mathrm{C}-\mathrm{X}_{23}-\mathrm{HXH}$ \\
\hline VvWRKY 3-1 & GI:48686707 & 1 & 317 & 7.64 & 58.3 & Vitis vinifera & $\begin{array}{l}\text { I-NTWD } \\
\text { I-CTWD }\end{array}$ & $\begin{array}{l}3-1 \mathrm{~N} \\
3-1 \mathrm{C}\end{array}$ & $\begin{array}{l}\mathrm{C}-\mathrm{X}_{4}-\mathrm{C}-\mathrm{X}_{22}-\mathrm{HXH} \\
\mathrm{C}-\mathrm{X}_{4}-\mathrm{C}-\mathrm{X}_{23}-\mathrm{HXH}\end{array}$ \\
\hline VvWRKY 3-2 & GI:315272006 & $*$ & 534 & 8.22 & 35.3 & Vitis vinifera & IIa & $3-2$ & $\mathrm{C}-\mathrm{X}_{5}-\mathrm{C}-\mathrm{X}_{23}-\mathrm{HXH}$ \\
\hline VvWRKY 4 & GI:315272008 & $*$ & 625 & 8.71 & 34.4 & Vitis vinifera & IIa & 4 & $\mathrm{C}-\mathrm{X}_{5}-\mathrm{C}-\mathrm{X}_{25}-\mathrm{HXH}$ \\
\hline VvWRKY 6-1 & GI:359485613 & 12 & 753 & 6.48 & 64.1 & Vitis vinifera & IIb & $6-1$ & $\mathrm{C}-\mathrm{X}_{5}-\mathrm{C}-\mathrm{X}_{23}-\mathrm{HXH}$ \\
\hline VvWRKY 6-2 & GI:225444291 & 10 & 535 & 6.04 & 57.5 & Vitis vinifera & IIb & $6-2$ & $\mathrm{C}-\mathrm{X}_{5}-\mathrm{C}-\mathrm{X}_{23}-\mathrm{HXH}$ \\
\hline VvWRKY 7-1 & GI:225458699 & 18 & 347 & 9.36 & 38.1 & Vitis vinifera & IId & $7-1$ & $\mathrm{C}-\mathrm{X}_{5}-\mathrm{C}-\mathrm{X}_{23}-\mathrm{HXH}$ \\
\hline VvWRKY 7-2 & GI:225438803 & 7 & 535 & 9.57 & 36.6 & Vitis vinifera & IId & $7-2$ & $\mathrm{C}-\mathrm{X}_{5}-\mathrm{C}-\mathrm{X}_{23}-\mathrm{HXH}$ \\
\hline VvWRKY 9 & GI:225447777 & 12 & 798 & 5.16 & 52.4 & Vitis vinifera & IIb & 9 & $\mathrm{C}-\mathrm{X}_{5}-\mathrm{C}-\mathrm{X}_{23}-\mathrm{HXH}$ \\
\hline VvWRKY 11-1 & GI:225466161 & 4 & 338 & 9.49 & 36.6 & Vitis vinifera & IId & $11-1$ & $\mathrm{C}-\mathrm{X}_{5}-\mathrm{C}-\mathrm{X}_{23}-\mathrm{HXH}$ \\
\hline VvWRKY 11-2 & GI:225445976 & 11 & 297 & 9.73 & 32.4 & Vitis vinifera & IId & $11-2$ & $\mathrm{C}-\mathrm{X}_{5}-\mathrm{C}-\mathrm{X}_{23}-\mathrm{HXH}$ \\
\hline VvWRKY 11-3 & GI:262091438 & $*$ & 297 & 9.73 & 32.4 & Vitis vinifera & IId & $1-3$ & $\mathrm{C}-\mathrm{X}_{5}-\mathrm{C}-\mathrm{X}_{23}-\mathrm{HXH}$ \\
\hline VvWRKY 12-1 & GI:225453346 & 15 & 228 & 7.68 & 26.1 & Vitis vinifera & IIc & $12-1$ & $\mathrm{C}-\mathrm{X}_{4}-\mathrm{C}-\mathrm{X}_{23}-\mathrm{HXH}$ \\
\hline VvWRKY 12-2 & GI:225453345 & 15 & 228 & 7.8 & 26.1 & Vitis vinifera & IIc & $12-2$ & $\mathrm{C}-\mathrm{X}_{4}-\mathrm{C}-\mathrm{X}_{23}-\mathrm{HXH}$ \\
\hline VvWRKY 13-1 & GI:359472522 & 1 & 305 & 5.8 & 33.4 & Vitis vinifera & IIc & $13-1$ & $\mathrm{C}-\mathrm{X}_{4}-\mathrm{C}-\mathrm{X}_{23}-\mathrm{HXH}$ \\
\hline VvWRKY 13-1 & GI:359472523 & 1 & 305 & 5.81 & 33.4 & Vitis vinifera & IIc & $13-1$ & $\mathrm{C}-\mathrm{X}_{4}-\mathrm{C}-\mathrm{X}_{23}-\mathrm{HXH}$ \\
\hline VvWRKY 14 & GI:225444177 & 10 & 438 & 5.16 & 47.5 & Vitis vinifera & IIe & 14 & $\mathrm{C}-\mathrm{X}_{5}-\mathrm{C}-\mathrm{X}_{23}-\mathrm{HXH}$ \\
\hline VvWRKY 18 & GI:359476150 & 4 & 261 & 9.1 & 28.9 & Vitis vinifera & IIa & 18 & $\mathrm{C}-\mathrm{X}_{5}-\mathrm{C}-\mathrm{X}_{23}-\mathrm{HXH}$ \\
\hline VvWRKY 20-1 & GI:359496861 & $*$ & 604 & 6.01 & 65.7 & Vitis vinifera & $\begin{array}{l}\text { I-NTWD } \\
\text { I-CTWD }\end{array}$ & $\begin{array}{l}20-1 \mathrm{~N} \\
20-1 \mathrm{C}\end{array}$ & $\begin{array}{l}\mathrm{C}-\mathrm{X}_{4}-\mathrm{C}-\mathrm{X}_{22}-\mathrm{HXH} \\
\mathrm{C}-\mathrm{X}_{4}-\mathrm{C}-\mathrm{X}_{23}-\mathrm{HXH}\end{array}$ \\
\hline VvWRKY 20-2 & GI:359494165 & 19 & 580 & 6.27 & 63.4 & Vitis vinifera & $\begin{array}{l}\text { I-NTWD } \\
\text { I-CTWD }\end{array}$ & $\begin{array}{l}20-2 \mathrm{~N} \\
20-2 \mathrm{C}\end{array}$ & $\begin{array}{l}\mathrm{C}-\mathrm{X}_{4}-\mathrm{C}-\mathrm{X}_{23}-\mathrm{HXH} \\
\mathrm{C}-\mathrm{X}_{4}-\mathrm{C}-\mathrm{X}_{23}-\mathrm{HXH}\end{array}$ \\
\hline VvWRKY 20-3 & GI:225447598 & 12 & 407 & 5.02 & 44.8 & Vitis vinifera & $\begin{array}{l}\text { I-NTWD } \\
\text { I-CTWD }\end{array}$ & $\begin{array}{l}20-3 \mathrm{~N} \\
20-3 \mathrm{C}\end{array}$ & $\begin{array}{l}\mathrm{C}-\mathrm{X}_{4}-\mathrm{C}-\mathrm{X}_{22}-\mathrm{HXH} \\
\mathrm{C}-\mathrm{X}_{4}-\mathrm{C}-\mathrm{X}_{23}-\mathrm{HXH}\end{array}$ \\
\hline VvWRKY 20-4 & GI:359496860 & 12 & 514 & 6.6 & 56.2 & Vitis vinifera & $\begin{array}{l}\text { I-NTWD } \\
\text { I-CTWD }\end{array}$ & $\begin{array}{l}20-4 \mathrm{~N} \\
20-4 \mathrm{C}\end{array}$ & $\begin{array}{l}\mathrm{C}-\mathrm{X}_{4}-\mathrm{C}-\mathrm{X}_{23}-\mathrm{HXH} \\
\mathrm{C}-\mathrm{X}_{4}-\mathrm{C}-\mathrm{X}_{23}-\mathrm{HXH}\end{array}$ \\
\hline VvWRKY 20-5 & GI:359494164 & 19 & 595 & 6.8 & 65 & Vitis vinifera & $\begin{array}{l}\text { I-NTWD } \\
\text { I-CTWD }\end{array}$ & $\begin{array}{l}20-5 \mathrm{~N} \\
20-5 \mathrm{C}\end{array}$ & $\begin{array}{l}\mathrm{C}-\mathrm{X}_{4}-\mathrm{C}-\mathrm{X}_{23}-\mathrm{HXH} \\
\mathrm{C}-\mathrm{X}_{4}-\mathrm{C}-\mathrm{X}_{22}-\mathrm{HXH}\end{array}$ \\
\hline VvWRKY 20-6 & GI:359485885 & 12 & 407 & 4.8 & 44.8 & Vitis vinifera & $\begin{array}{l}\text { I-NTWD } \\
\text { I-CTWD }\end{array}$ & $\begin{array}{l}20-6 N \\
20-6 C\end{array}$ & $\begin{array}{l}\mathrm{C}-\mathrm{X}_{4}-\mathrm{C}-\mathrm{X}_{23}-\mathrm{HXH} \\
\mathrm{C}-\mathrm{X}_{4}-\mathrm{C}-\mathrm{X}_{23}-\mathrm{HXH}\end{array}$ \\
\hline VvWRKY 21 & GI:225437249 & 7 & 340 & 9.84 & 38 & Vitis vinifera & IId & 21 & $\mathrm{C}-\mathrm{X}_{5}-\mathrm{C}-\mathrm{X}_{23}-\mathrm{HXH}$ \\
\hline VvWRKY 22-1 & GI:359480165 & 7 & 233 & 6.06 & 26.8 & Vitis vinifera & IIe & $22-1$ & $\mathrm{C}-\mathrm{X}_{5}-\mathrm{C}-\mathrm{X}_{23}-\mathrm{HXH}$ \\
\hline VvWRKY 22-2 & GI:225454298 & 15 & 348 & 5.73 & 38 & Vitis vinifera & IIe & $22-2$ & $\mathrm{C}-\mathrm{X}_{5}-\mathrm{C}-\mathrm{X}_{23}-\mathrm{HXH}$ \\
\hline VvWRKY 22-3 & GI:225426142 & 2 & 331 & 5.71 & 36.6 & Vitis vinifera & IIe & $22-3$ & $\mathrm{C}-\mathrm{X}_{5}-\mathrm{C}-\mathrm{X}_{23}-\mathrm{HXH}$ \\
\hline VvWRKY 22-4 & GI:225464629 & * & 166 & 4.7 & 18.4 & Vitis vinifera & IIe & $22-4$ & $\mathrm{C}-\mathrm{X}_{5}-\mathrm{C}-\mathrm{X}_{23}-\mathrm{HXH}$ \\
\hline VvWRKY 23 & GI:225437606 & 7 & 302 & 6.74 & 33.8 & Vitis vinifera & IIc & 23 & $\mathrm{C}-\mathrm{X}_{4}-\mathrm{C}-\mathrm{X}_{23}-\mathrm{HXH}$ \\
\hline VvWRKY 24 & GI:359489647 & 15 & 165 & 9.61 & 19 & Vitis vinifera & IIc & 24 & $\mathrm{C}-\mathrm{X}_{4}-\mathrm{C}-\mathrm{X}_{23}-\mathrm{HXH}$ \\
\hline VvWRKY 28-1 & GI:225463412 & 10 & 319 & 6.76 & 35 & Vitis vinifera & IIc & $28-1$ & $\mathrm{C}-\mathrm{X}_{4}-\mathrm{C}-\mathrm{X}_{23}-\mathrm{HXH}$ \\
\hline VvWRKY 28-2 & GI:225446835 & 12 & 311 & 6.92 & 34.8 & Vitis vinifera & IIc & $28-2$ & $\mathrm{C}-\mathrm{X}_{4}-\mathrm{C}-\mathrm{X}_{23}-\mathrm{HXH}$ \\
\hline VvWRKY 30 & GI:40846374 & * & 349 & 5.02 & 39.3 & Vitis aestivalis & III & 30 & $\mathrm{C}-\mathrm{X}_{7}-\mathrm{C}-\mathrm{X}_{23}-\mathrm{HTC}$ \\
\hline
\end{tabular}


TABle 1: Continued.

\begin{tabular}{|c|c|c|c|c|c|c|c|c|c|}
\hline \multirow{2}{*}{ Name } & \multirow{2}{*}{ Protein $^{a}$} & \multirow{2}{*}{ Chr } & \multicolumn{3}{|c|}{ Deduced polypeptide } & \multirow{2}{*}{ Organism } & \multirow{2}{*}{ Group } & \multicolumn{2}{|c|}{ Domain } \\
\hline & & & Length (aa) & PI & MW (kDa) & & & Family name & Pattern \\
\hline \multirow{2}{*}{ VvWRKY 32-1 } & \multirow{2}{*}{ GI:225445873 } & \multirow{2}{*}{11} & \multirow{2}{*}{499} & \multirow{2}{*}{6.12} & \multirow{2}{*}{53.8} & \multirow{2}{*}{ Vitis vinifera } & I-NTWD & $32-1 \mathrm{~N}$ & $\mathrm{C}-\mathrm{X}_{4}-\mathrm{C}-\mathrm{X}_{22}-\mathrm{HXH}$ \\
\hline & & & & & & & I-CTWD & $32-1 \mathrm{C}$ & $\mathrm{C}-\mathrm{X}_{4}-\mathrm{C}-\mathrm{X}_{23}-\mathrm{HXH}$ \\
\hline \multirow{2}{*}{ VvWRKY 32-2 } & \multirow{2}{*}{ GI:225430477 } & \multirow{2}{*}{4} & \multirow{2}{*}{475} & \multirow{2}{*}{8.47} & 526 & Vitis vinit & I-NTWD & $32-2 \mathrm{~N}$ & $\mathrm{C}-\mathrm{X}_{4}-\mathrm{C}-\mathrm{X}_{22}-\mathrm{HXH}$ \\
\hline & & & & & 32.0 & vilo & I-CTWD & $32-2 \mathrm{C}$ & $\mathrm{C}-\mathrm{X}_{4}-\mathrm{C}-\mathrm{X}_{23}-\mathrm{HXH}$ \\
\hline VyWRKY 33-1 & GI.225439574 & 8 & 552 & 729 & 61 & & I-NTWD & $33-1 \mathrm{~N}$ & $\mathrm{C}-\mathrm{X}_{4}-\mathrm{C}-\mathrm{X}_{22}-\mathrm{HXH}$ \\
\hline VVVRK K & & 8 & 352 & 1.29 & 01 & & I-CTWD & $33-1 C$ & $\mathrm{C}-\mathrm{X}_{4}-\mathrm{C}-\mathrm{X}_{23}-\mathrm{HXH}$ \\
\hline & & & 603 & & & & I-NTWD & $33-2 \mathrm{~N}$ & $\mathrm{C}-\mathrm{X}_{4}-\mathrm{C}-\mathrm{X}_{22}-\mathrm{HXH}$ \\
\hline V КKY 33-2 & G1:225434421 & 6 & 603 & 0.4 & 66.3 & & I-CTWD & $33-2 \mathrm{C}$ & $\mathrm{C}-\mathrm{X}_{4}-\mathrm{C}-\mathrm{X}_{23}-\mathrm{HXH}$ \\
\hline VvWRKY 40-1 & GI:225443178 & 9 & 311 & 5.16 & 52.4 & Vitis vinifera & IIa & $40-1$ & $\mathrm{C}-\mathrm{X}_{5}-\mathrm{C}-\mathrm{X}_{25}-\mathrm{HXH}$ \\
\hline VvWRKY 40-2 & GI:225430340 & 4 & 317 & 8.22 & 35.3 & Vitis vinifera & IIa & $40-2$ & $\mathrm{C}-\mathrm{X}_{5}-\mathrm{C}-\mathrm{X}_{23}-\mathrm{HXH}$ \\
\hline VvWRKY 41 & GI:225426000 & 2 & 342 & 6.05 & 38.6 & Vitis vinifera & III & 41 & $\mathrm{C}-\mathrm{X}_{7}-\mathrm{C}-\mathrm{X}_{23}-\mathrm{HTC}$ \\
\hline VvWRKY 42 & GI:359494147 & 19 & 511 & 9.2 & 55.1 & Vitis vinifera & IIb & 42 & $\mathrm{C}-\mathrm{X}_{5}-\mathrm{C}-\mathrm{X}_{23}-\mathrm{HXH}$ \\
\hline VyWRKY 44 & 439779 & & 477 & 8.84 & 52.3 & & I-NTWD & $44 \mathrm{C}$ & $\mathrm{C}-\mathrm{X}_{4}-\mathrm{C}-\mathrm{X}_{22}-\mathrm{HXH}$ \\
\hline VVWRK & $439 / / 9$ & 8 & $4 / /$ & 8.84 & 52.3 & & I-CTWD & $44 \mathrm{~N}$ & $\mathrm{C}-\mathrm{X}_{4}-\mathrm{C}-\mathrm{X}_{23}-\mathrm{HXH}$ \\
\hline VvWRKY 45 & GI:225451489 & 14 & 182 & 9.41 & 20.8 & Vitis vinifera & IIc & 45 & $\mathrm{C}-\mathrm{X}_{4}-\mathrm{C}-\mathrm{X}_{23}-\mathrm{HXH}$ \\
\hline VvWRKY 46 & GI:225454483 & 15 & 349 & 5.01 & 39.2 & Vitis vinifera & III & 46 & $\mathrm{C}-\mathrm{X}_{7}-\mathrm{C}-\mathrm{X}_{23}-\mathrm{HTC}$ \\
\hline VvWRKY 47 & GI:225437767 & 7 & 505 & 8.03 & 54.7 & Vitis vinifera & $\mathrm{IIb}$ & 47 & $\mathrm{C}-\mathrm{X}_{5}-\mathrm{C}-\mathrm{X}_{23}-\mathrm{HXH}$ \\
\hline VvWRKY 48 & GI:225432004 & 5 & 309 & 5.72 & 34.4 & Vitis vinifera & IIc & 48 & $\mathrm{C}-\mathrm{X}_{4}-\mathrm{C}-\mathrm{X}_{23}-\mathrm{HXH}$ \\
\hline VvWRKY 49 & GI:225440394 & 8 & 299 & 5.16 & 52.4 & Vitis vinifera & IIc & 49 & $\mathrm{C}-\mathrm{X}_{4}-\mathrm{C}-\mathrm{X}_{23}-\mathrm{HXH}$ \\
\hline VvWRKY 50 & GI:225429590 & 4 & 166 & 5.2 & 18.9 & Vitis vinifera & IIc & 50 & $\mathrm{C}-\mathrm{X}_{4}-\mathrm{C}-\mathrm{X}_{23}-\mathrm{HXH}$ \\
\hline VvWRKY 51-1 & GI:359476460 & 4 & 136 & 9.39 & 15.8 & Vitis vinifera & IIc & $51-1$ & $\mathrm{C}-\mathrm{X}_{4}-\mathrm{C}-\mathrm{X}_{23}-\mathrm{HXH}$ \\
\hline VvWRKY 51-2 & GI:359480857 & 7 & 149 & 9.07 & 17.2 & Vitis vinifera & IIc & $51-2$ & $\mathrm{C}-\mathrm{X}_{4}-\mathrm{C}-\mathrm{X}_{23}-\mathrm{HXH}$ \\
\hline VvWRKY 51-3 & GI:225466167 & 4 & 191 & 5.58 & 21.5 & Vitis vinifera & IIc & $51-3$ & $\mathrm{C}-\mathrm{X}_{4}-\mathrm{C}-\mathrm{X}_{23}-\mathrm{HXH}$ \\
\hline VvWRKY 51-4 & GI:359480856 & 7 & 193 & 7.1 & 21.5 & Vitis vinifera & IIc & $51-4$ & $\mathrm{C}-\mathrm{X}_{4}-\mathrm{C}-\mathrm{X}_{23}-\mathrm{HXH}$ \\
\hline VvWRKY 53 & GI:359490533 & 16 & 364 & 5.45 & 40 & Vitis vinifera & III & 53 & $\mathrm{C}-\mathrm{X}_{7}-\mathrm{C}-\mathrm{X}_{23}-\mathrm{HTC}$ \\
\hline VvWRKY 57-1 & GI:225423515 & 1 & 305 & 5.62 & 33.4 & Vitis vinifera & IIc & $57-1$ & $\mathrm{C}-\mathrm{X}_{4}-\mathrm{C}-\mathrm{X}_{23}-\mathrm{HXH}$ \\
\hline VvWRKY 57-2 & GI:225425363 & 1 & 189 & 9.4 & 21.3 & Vitis vinifera & IIc & $57-2$ & $\mathrm{C}-\mathrm{X}_{4}-\mathrm{C}-\mathrm{X}_{23}-\mathrm{HXH}$ \\
\hline VvWRKY 65-1 & GI:225443744 & 10 & 278 & 5.14 & 3.9 & Vitis vinifera & IIe & $65-1$ & $\mathrm{C}-\mathrm{X}_{5}-\mathrm{C}-\mathrm{X}_{23}-\mathrm{HXH}$ \\
\hline VvWRKY 65-2 & GI:225446682 & 12 & 244 & 5.41 & 26.4 & Vitis vinifera & IIe & $65-2$ & $\mathrm{C}-\mathrm{X}_{5}-\mathrm{C}-\mathrm{X}_{23}-\mathrm{HXH}$ \\
\hline VvWRKY 65-3 & GI:359485307 & 12 & 244 & 5.4 & 26.4 & Vitis vinifera & IIe & $65-3$ & $\mathrm{C}-\mathrm{X}_{5}-\mathrm{C}-\mathrm{X}_{23}-\mathrm{HXH}$ \\
\hline VvWRKY 55 & GI:225448719 & 13 & 364 & 5.97 & 40.3 & Vitis vinifera & III & 55 & $\mathrm{C}-\mathrm{X}_{7}-\mathrm{C}-\mathrm{X}_{23}-\mathrm{HTC}$ \\
\hline VvWRKY 70-1 & GI:225448721 & 13 & 313 & 5.45 & 35.3 & Vitis vinifera & III & $70-1$ & $\mathrm{C}-\mathrm{X}_{7}-\mathrm{C}-\mathrm{X}_{23}-\mathrm{HTC}$ \\
\hline VvWRKY 70-2 & GI:225439707 & 8 & 322 & 5.49 & 36.6 & Vitis vinifera & III & $70-2$ & $\mathrm{C}-\mathrm{X}_{7}-\mathrm{C}-\mathrm{X}_{23}-\mathrm{HTC}$ \\
\hline VvWRKY 72-1 & GI:359491334 & 17 & 611 & 7.9 & 65.7 & Vitis vinifera & IIb & $72-2$ & $\mathrm{C}-\mathrm{X}_{5}-\mathrm{C}-\mathrm{X}_{23}-\mathrm{HXH}$ \\
\hline VvWRKY 72-2 & GI:359488978 & 14 & 755 & 5.85 & 59.7 & Vitis vinifera & $\mathrm{IIb}$ & $72-2$ & $\mathrm{C}-\mathrm{X}_{5}-\mathrm{C}-\mathrm{X}_{23}-\mathrm{HXH}$ \\
\hline VvWRKY 72-3 & GI:359473376 & 1 & 547 & 5.92 & 59.7 & Vitis vinifera & $\mathrm{IIb}$ & $72-3$ & $\mathrm{C}-\mathrm{X}_{5}-\mathrm{C}-\mathrm{X}_{23}-\mathrm{HXH}$ \\
\hline VvWRKY 74 & GI:225463956 & 14 & 362 & 9.68 & 41.3 & Vitis vinifera & IId & 74 & $\mathrm{C}-\mathrm{X}_{5}-\mathrm{C}-\mathrm{X}_{23}-\mathrm{HXH}$ \\
\hline VvWRKY 75 & GI:225456341 & 17 & 151 & 9.67 & 17.7 & Vitis vinifera & IIc & 75 & $\mathrm{C}-\mathrm{X}_{4}-\mathrm{C}-\mathrm{X}_{23}-\mathrm{HXH}$ \\
\hline VtWRKY5 & GJ·183979104 & $*$ & 529 & 772 & 577 & Vit & I-NTWD & $\mathrm{Vt} 5 \mathrm{~N}$ & $\mathrm{C}-\mathrm{X}_{4}-\mathrm{C}-\mathrm{X}_{22}-\mathrm{HXH}$ \\
\hline & & & & & & & I-CTWD & $\mathrm{Vt5C}$ & $\mathrm{C}-\mathrm{X}_{4}-\mathrm{C}-\mathrm{X}_{23}-\mathrm{HXH}$ \\
\hline VtWRKY7 & GI:183979108 & * & 603 & 6.42 & 66.3 & & I-NTWD & Vt7N & $\mathrm{C}-\mathrm{X}_{4}-\mathrm{C}-\mathrm{X}_{22}-\mathrm{HXH}$ \\
\hline 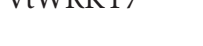 & 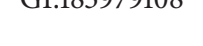 & ${ }^{*}$ & (00J & & & & I-CTWD & Vt7C & $\mathrm{C}-\mathrm{X}_{4}-\mathrm{C}-\mathrm{X}_{23}-\mathrm{HXH}$ \\
\hline VtWRKY11 & GI:183979106 & $*$ & 338 & 9.49 & 36.6 & Vitis thunbergii & IId & Vt11 & $\mathrm{C}-\mathrm{X}_{5}-\mathrm{C}-\mathrm{X}_{23}-\mathrm{HXH}$ \\
\hline VaWRKY4 & GI:40060529 & * & 311 & 8.71 & 34.4 & Vitis aestivalis & IIa & $\mathrm{Va} 4$ & $\mathrm{C}-\mathrm{X}_{5}-\mathrm{C}-\mathrm{X}_{23}-\mathrm{HXH}$ \\
\hline VpWRKY1 & GI:263199372 & * & 322 & 5.58 & 36.5 & Vitis pseudoreticulata & III & Vp1 & $\mathrm{C}-\mathrm{X}_{7}-\mathrm{C}-\mathrm{X}_{23}-\mathrm{HTC}$ \\
\hline VpWRKY2 & GI:290894627 & * & 499 & 6.23 & 53.9 & & I-NTWD & $\mathrm{Vp} 2 \mathrm{~N}$ & $\mathrm{C}-\mathrm{X}_{4}-\mathrm{C}-\mathrm{X}_{22}-\mathrm{HXH}$ \\
\hline & & $*$ & & $0.2 J$ & & ala & I-CTWD & Vp2C & $\mathrm{C}-\mathrm{X}_{4}-\mathrm{C}-\mathrm{X}_{23}-\mathrm{HXH}$ \\
\hline VpWRKY3 & GI:345104746 & * & 319 & 7.67 & 35.5 & Vitis pseudoreticulata & IIa & Vp3 & $\mathrm{C}-\mathrm{X}_{5}-\mathrm{C}-\mathrm{X}_{23}-\mathrm{HXH}$ \\
\hline VbcWRKY50 & GI:163914201 & * & 127 & 9.4 & 14.3 & Vitis hybrid cultivar & IId & Vbc50 & $\mathrm{C}-\mathrm{X}_{5}-\mathrm{C}-\mathrm{X}_{23}-\mathrm{HXH}$ \\
\hline
\end{tabular}

${ }^{\mathrm{a}}$ GenBank protein number

Chr.: chromosome; ORF: open reading frame; ${ }^{*}$ chromosome unknown. 


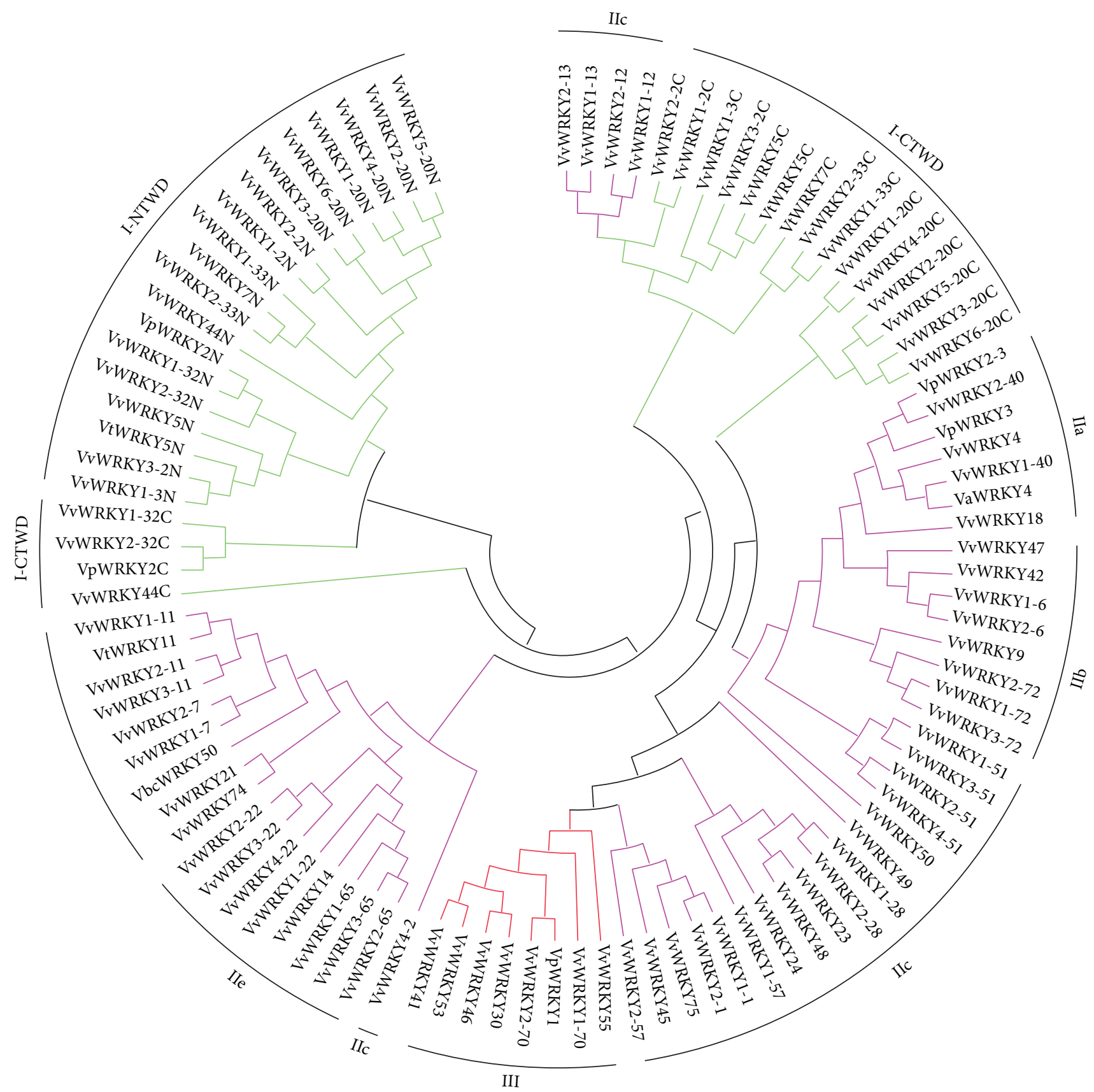

FIGURE 2: Phylogenetic tree based on amino acid sequences as determined by the MEGA ver4.0 software using the neighbor-joining method. Bootstrap values $(\geq 500)$ based on 1,000 replications are exhibited beside the nodes.

domain) members, VvWRKY32-1C, VvWRKY32-2C, and VpWRKY2C, were more closely related to the N-terminal members of Group I than to the other C-terminal members (Figures 2, and 3). While Group I VvWRKY44C was far away from both I-CTWD and N-terminal, and unique, which suggesting a special origin of the domain. The largest number of WRKY proteins of Group II was divided to five among 23 major subgroups: IIa, IIb, IIc, IId, and IIe. Group IIa (seven members) and Group IIb (eight members) were two subgroups from the same branch, while Group
IId (nine members) and Group IIe (eight members) were close in genetic tree. Five members of Group IIc were more similar to Groups IIa and IIb from the same branch based on the phylogenetic analysis. Four members of Group IIc, VvWRKY13-1, 13-2, 12-1, and 12-2, were more closely related to the C-terminal WRKY domains of Group I than those of other groups. This is consistent with a recent analysis supporting the hypothesis that loss of the Group I N-terminal WRKY domain led to the origin of the Group II WRKY proteins [5]. 


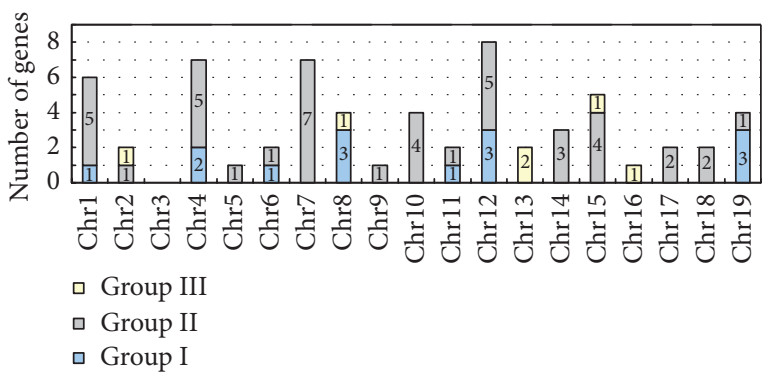

FIGURE 3: Histogram of the number and distribution of three groups of WRKY genes on 19 chromosomes.

Seven WRKY domains belonged to Group III, which is generally considered to be the most evolutionarily advanced group and the most adaptable [5]. Phylogenetic relationships between the Group III WRKY domains of 15 AtWRKY, 28 OsWRKY, 10 PtWRKY, and 7 VvWRKY proteins were examined (Figure 4). The poplar WRKY domains shared higher sequence homology with the other dicot plants (Arabidopsis and grape) than with monocot rice. Similarly, the six AtWRKY domains clustered in two groups (Figure 4). However, this diversity was not found in Arabidopsis, poplar, or grape, suggesting that VvWRKY genes respond to different environmental signals.

3.3. Conserved Motifs and Domains in Grape WRKY Proteins. With the exception of the conserved 60 amino acid residues, no functional or structural homologies were present in the WRKY protein sequences [7]. Few WRKY proteins contain a conserved leucine zipper motif, a hypothetical structure common to a class of DNA-binding proteins [7, 32]. Using the online 2ZIP software, we found that none of the grape WRKY proteins contained this structure with the exception of VvWRKY18. The conserved HARF sequence motif (RTGHARFRR(A/G)P) was found in six members (VvWRKY7-1, -7-2,-11-1, -11-2, -11-3, and VtWRKY11) of the Group IId WRKY proteins in Arabidopsis, although no putative function has been clearly identified for this motif $[7,33]$. WRKY proteins are transcription factors associated with activation and repression of plant immune responses $[7,10,34]$. The coactivator motif, LXXLL (L, leucine; X, any amino acid), and the active repressor motif, LXLXLX $[35,36]$, were searched for in the VvWRKY protein sequences by manual inspection. Seven VvWRKY proteins, VpWRKY3 and VvWRKY20-3, -42, -9, -18, -3-2, and -40-2, contained the LXLXLX motif and three VvWRKY proteins (VvWRKY72, -11-2, and 11-3) contained the LXXLL motif. The Multiple expectation maximization for motif elicitation online software was used as a secondary method to analyze motif distribution and confirm the results of domain prediction (Figure 5; Table 2). LZ indicates potential leucine zipper structures that were also predicted by the COILSCAN and COIL programs but none was found. The conserved motifs $1,2,3,4,5$, and 6 in Figure 5 were characterized as WRKY domains and were broadly distributed among the VxWRKY protein sequences. Motif 10 and motif 13, a conserved NLS motif, were found mainly among the Groups I and IId proteins, while motif 12 was found only among Groups I and IId proteins, although its function is unknown.

3.4. Quantitative RT-PCR Analysis of Expression of $28 \mathrm{WRKY}$ Genes. To analyze expression patterns of WRKY genes during plant defense responses, we analyzed the expression profiles of 28 WRKY genes under disease conditions and in response to $\mathrm{SA}$ treatment using quantitative RT-PCR (Figure 6). Many of the WRKY genes exhibited significant changes in their expression levels in response to disease or SA. We isolated total RNA from leaves at various time points after infection with the fungal pathogen Coniothyrium diplodiella or after treatment with SA. The expression patterns of the 28 WRKY genes ( $v v W R K Y 1-1, V v W R K Y 2-1, V v W R K Y 3$, VvWRKY6-1, VvWRKY7-1, VvWRKY11-4, VvWRKY14, VvWRKY18, VvWRKY22-1, VvWRKY28, VvWRKY30, VvWRKY32, VvWRKY40, VvWRKY41, VvWRKY42, VvWRKY45, VvWRVvKY46, VvWRKY48, VvWRKY51, VvWRKY53, VvWRKY55, VvWRKY65, VvWRKY70-1, $V v W R K Y 70-2, W R K Y 72$, and VvWRKY74) were determined. After pathogen infection, three WRKY genes ( $V v W R K Y 48$, $V v W R K Y 51$, and VvWRKY45) showed little or no change in transcript levels, but the other 25 genes showed altered expression patterns. Among the 25 differentially regulated genes, the expression of two ( $V v W R K Y 3$ and $V v W R K Y 41)$ was repressed and that of 23 was induced. After SA treatment, 5 genes (VvWRKY41, VvWRKY30, VvWRKY42, VvWRKY46, and $V v W R K Y 70-1)$ showed little or no change in transcript levels, but expression of the other 23 genes was induced and upregulated. 16 of 23 (VvWRKY1-1, VvWRKY2-1, VvWRKY3, VvWRKY6-1, VvWRKY7-1, VvWRKY11-4, VvWRKY14, VvWRKY22-1, VvWRKY28, VvWRKY32, VvWRKY40, VvWRKY53, VvWRKY55, VvWRKY65, VvWRKY70-1, $V v W R K Y 70-2, W R K Y 72$, and VvWRKY74) upregulated $V v W R K Y$ genes were induced and upregulated by both pathogen infection and SA treatment (Figure 6).

\section{Discussion}

The WRKY transcription factor gene family appears to be involved in the regulation of a variety of processes [7, 21, 37]. The complex features and functions of this family have been studied extensively in the model herbaceous plants Arabidopsis and rice and in the woody plant poplar. Characterization of the WRKY genes in grape (Vitis spp.) would facilitate a broader understanding of this gene superfamily. In this study, 80 WRKY genes were characterized. The lengths of these sequences were highly varied implying a high degree of complexity among the $V v W R K Y$ genes.

The WRKY genes can be divided into three main groups based on their structural features. The Groups Ib and II $W R K Y$ genes are likely to have evolved from Group Ia WRKY genes through loss of the N-terminal WRKY domain. Replacement of the conserved His residue in the metalchelating zinc finger motif with a Cys residue may have led to the evolution of Group III WRKY genes [33]. The similar 


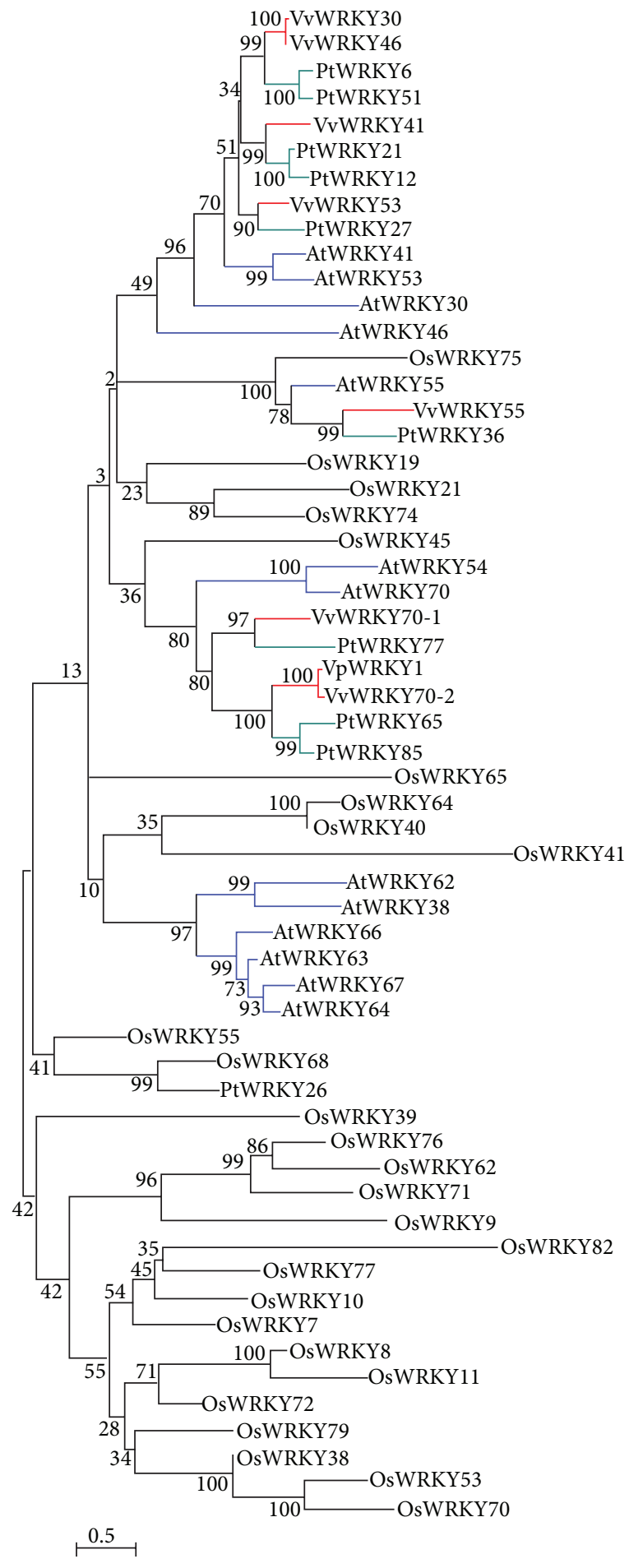

Figure 4: Phylogram of Group III WRKY domains from Arabidopsis (AtWRKY), rice (OsWRKY), poplar (PtWRKY), and grape (VvWRKY and VpWRKY). The alignment of amino acid sequences was produced using the MEGA ver4.0 program with the neighbor-joining method.

numbers of Arabidopsis, rice, poplar, and grape WRKY genes in Groups IIa and IIb suggest that all VvWRKY genes belonging to these subgroups have been identified, but there were only Group Ia in grape (Table 3). Fewer Group III $W R K Y$ genes have been identified in grape compared to in Arabidopsis and rice, close to the poplar quantitatively, implying that the number of $V v W R K Y$ genes in this group has either declined over evolutionary time or was underestimated in our analysis. Of the VvWRKY genes, $67.9 \%$ belong to Group II and 9.7\% to Group III. This distribution is more 

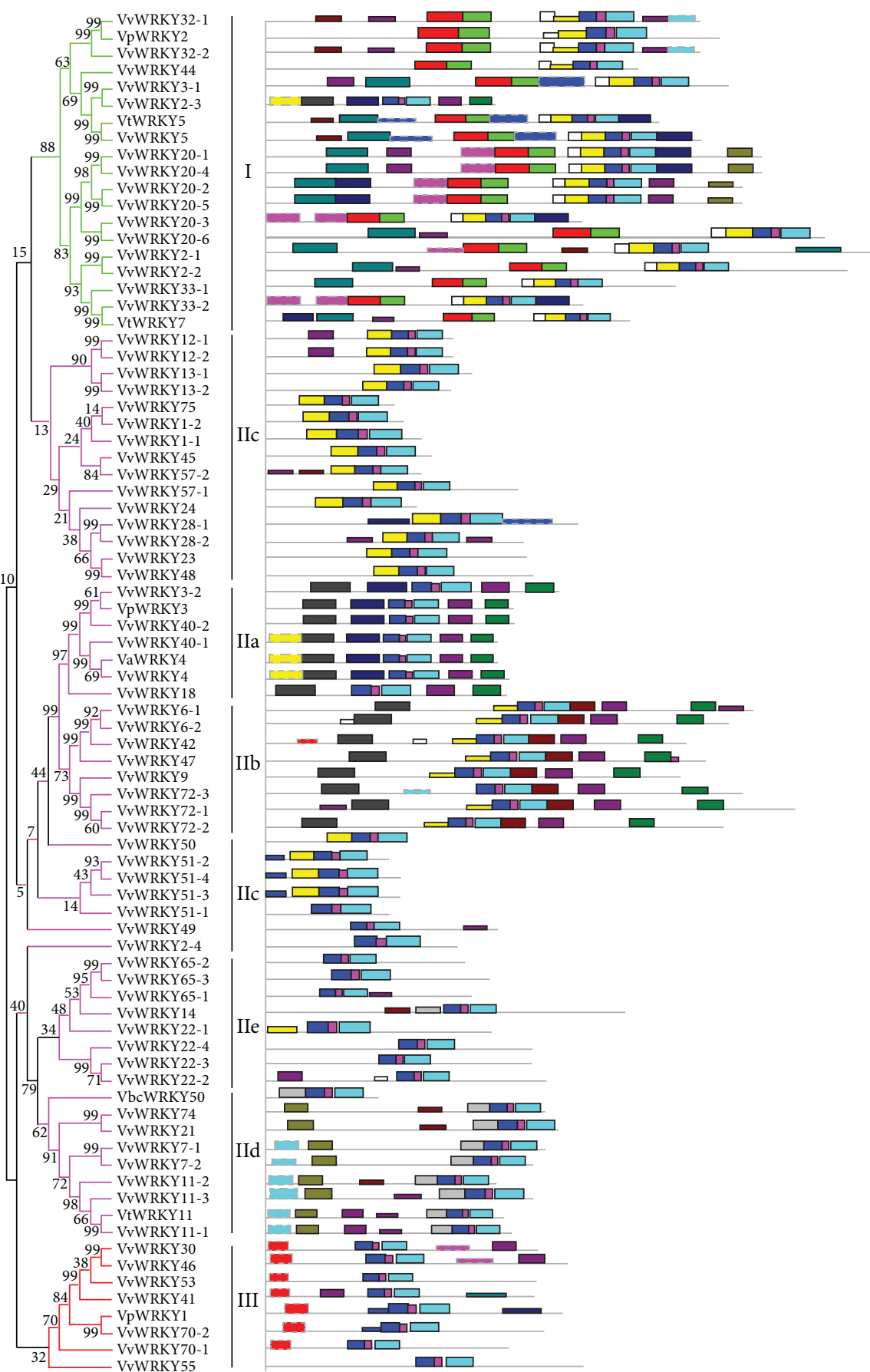

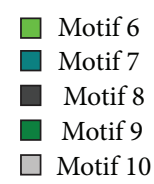

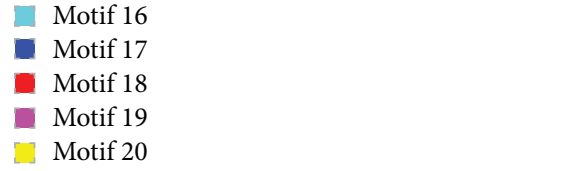

FIGURE 5: Phylogenetic analysis of 80 members of the $V v$ WRKY family. Amino acid sequences from the single WRKY domain of Groups II and III proteins or the C-terminal WRKY domain of Group I proteins were aligned. Conserved primary structure features of the $V v W R K Y$ family located outside the WRKY domains were identified using MEME software (http://www.sdsc.edu/MEME) and are shown below the tree. Schematic representations of typical proteins of each (sub) group are shown on the bottom; motifs of amino acid sequences were shown in Table 2; LZ indicates potential leucine zipper structures that were also predicted by the COILSCAN and COIL (Wisconsin Package Version 10.0) programs. 


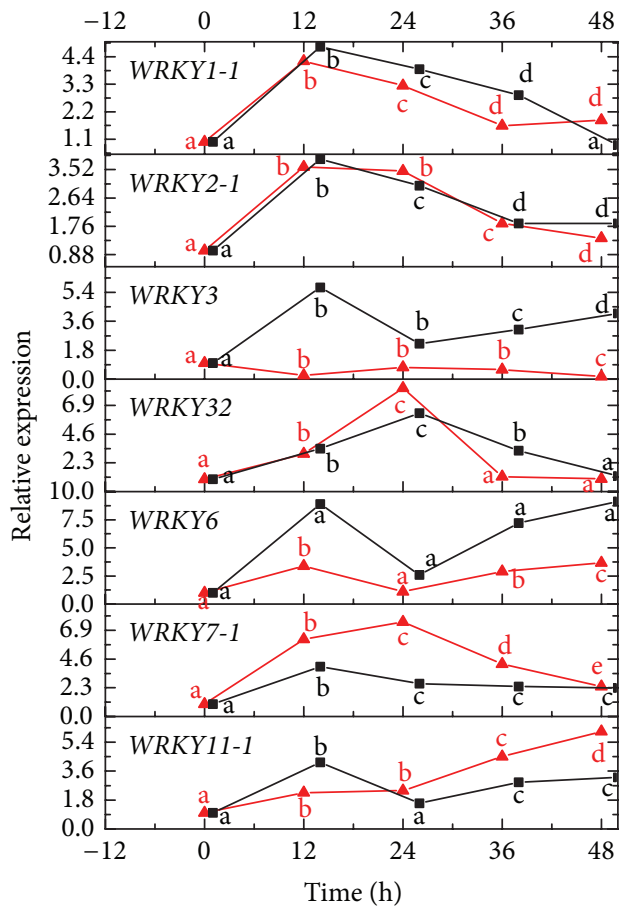

(a)

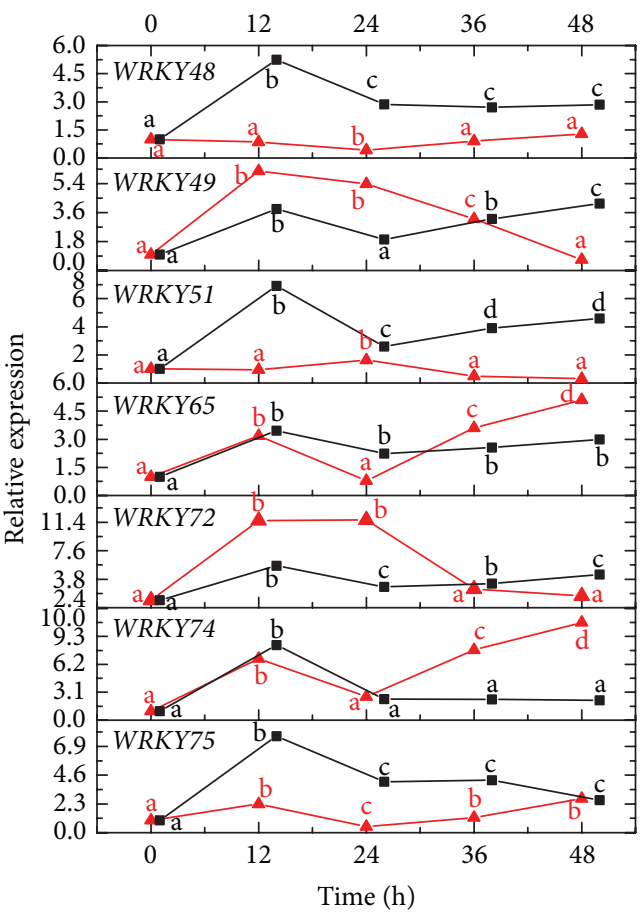

$\rightarrow$ White rot pathogenic bacteria $\rightarrow$ SA

(c)

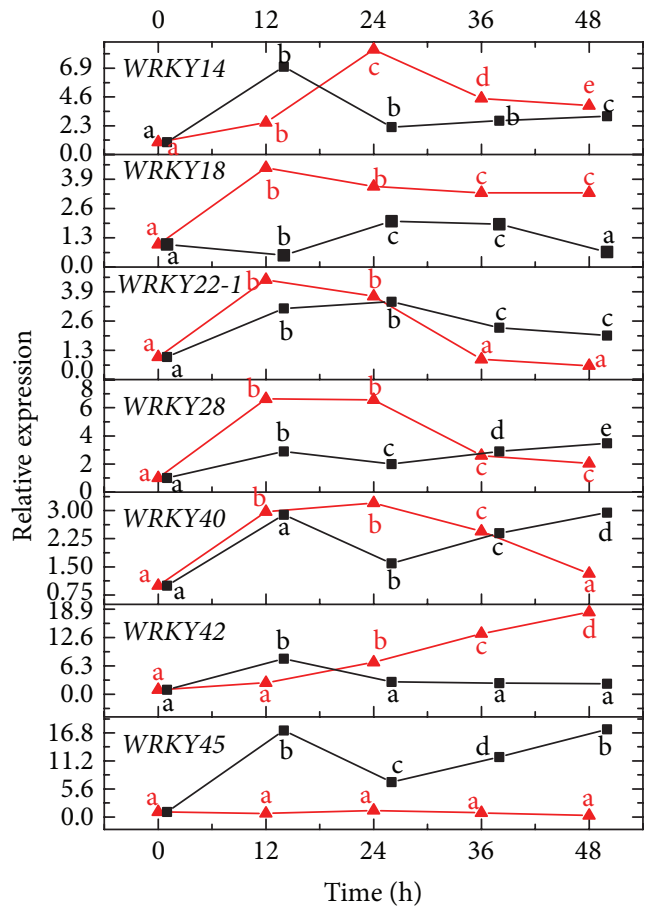

(b)

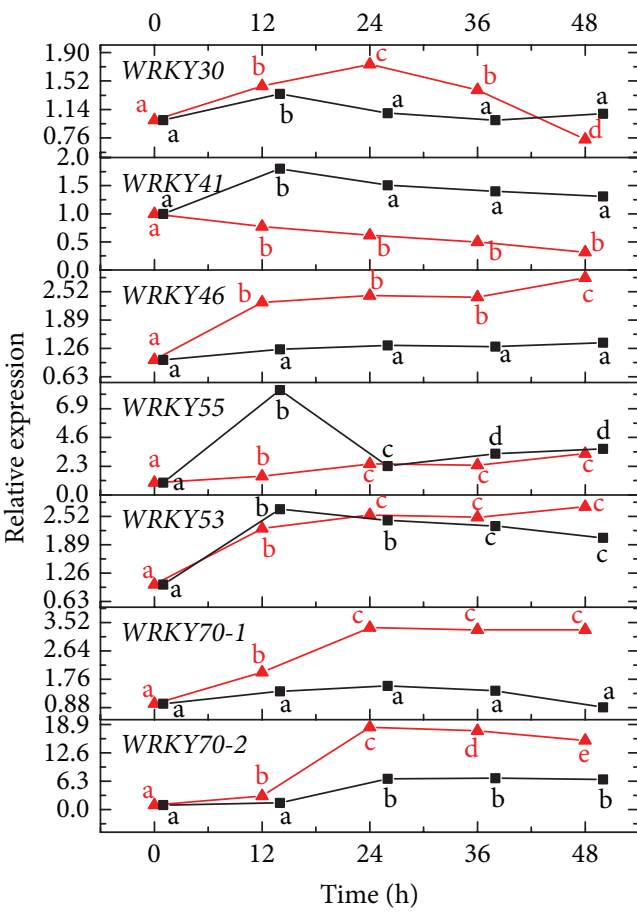

$\neg$ White rot pathogenic bacteria

$\rightarrow$ SA

(d)

FIGURE 6: Quantitative RT-PCR analysis of VdWRKY subgroup gene expression in response to pathogenic fungal infection and SA treatment. $0 \mathrm{~h}$ (control) indicates treatment with deionized water. Grape EF $\gamma$ gene (accession number AF176496) expression served as an internal control. 
TABLE 2: Motif sequences.

\begin{tabular}{|c|c|c|}
\hline Motif & Width & Best possible match \\
\hline 1 & 31 & GCPVRKHVERCSEDPSMVITTYEGEHNHPVP \\
\hline 2 & 21 & ILDDGYRWRKYGQKVIKGNPY \\
\hline 3 & 41 & EKPSEDGYNWRKYGQKQVKGSEYPRSYYKCTHPNCPVKKKV \\
\hline 4 & 8 & PRSYYRCT \\
\hline 5 & 29 & EEINKKDKKKGHKKIREPRFCFQTRSEVD \\
\hline 6 & 31 & ERSHDGQITEIIYKGTHNHPKPQPNRRYAVG \\
\hline 7 & 50 & YRQMRPAKLPIARSPCFTIPPGLSPTCLLDSPVFLSNMKVEPSPTTGTFP \\
\hline 8 & 41 & ETGVLVEELNRMNEENKKLREMLEIMCENYNALQMHLMELM \\
\hline 9 & 29 & FLVEQMTAAITKDPNFTAALAAAISGIIL \\
\hline 10 & 29 & SGRCHCSKRRKMRVKRTIRVPAISSKIAD \\
\hline 11 & 29 & QMASMMCPISMSTPFPTITLDLTKPTSFS \\
\hline 12 & 29 & INHFDCREITDYTVSKFKRVISILNRTGH \\
\hline 13 & 41 & NRNNIHGSVGNNTYSTSMDDIFRKKREETDKIKFRRVYYIT \\
\hline 14 & 29 & PAATAMASTTSAAASMLLSGSMTSQDGLM \\
\hline 15 & 15 & GDEDDEDEPDSKRWK \\
\hline 16 & 29 & GFSKMDEQIAIQEAASAGLKSMEHLIRLL \\
\hline 17 & 50 & DPNGHANFQENPELGSQGQMGNLNKPNEGLPAYSLPGMDQETTQAMPLHL \\
\hline 18 & 24 & WEHKTLINELTQGREMAKQLKIHL \\
\hline 19 & 41 & TSMESVPIEVDYDKLQQRQHFNIGVQASQSEQKETNPIIVV \\
\hline 20 & 41 & NWMAASLDLNANPLRLFDDTPKKEVQDDFTGLGLKVVSLKE \\
\hline
\end{tabular}

TABLE 3: Numbers of the various types of WRKY genes in Arabidopsis, rice, poplar, and grape.

\begin{tabular}{|c|c|c|c|c|c|c|}
\hline \multirow{2}{*}{ Group } & \multirow{2}{*}{ Subgroup } & \multicolumn{5}{|c|}{ Gene number } \\
\hline & & AtWRKY $^{\mathrm{a}}$ & OsWRKY $^{\mathrm{a}}$ & PtWRKY $^{\mathrm{a}}$ & VvWRKY $^{\mathrm{a}}$ & VxWRKY $^{\mathrm{b}}$ \\
\hline \multirow{3}{*}{ I } & & 32 & 34 & 50 & 16 & 3 \\
\hline & Ia & 14 & 14 & 23 & 16 & 3 \\
\hline & $\mathrm{Ib}$ & 18 & 20 & 27 & & \\
\hline \multirow{6}{*}{ II } & & 26 & 30 & 44 & & \\
\hline & IIa & 3 & 4 & 5 & 5 & 2 \\
\hline & IIb & 8 & 8 & 9 & 7 & \\
\hline & IIc & 7 & 7 & 13 & 22 & \\
\hline & IId & 8 & 11 & 13 & 7 & 2 \\
\hline & IIe & & & 4 & 8 & \\
\hline III & & 14 & 36 & 10 & 7 & 1 \\
\hline Total & & 72 & 100 & 104 & 72 & 8 \\
\hline
\end{tabular}

${ }^{\mathrm{a}}$ According to Wu et al. [20] and He et al. [21].

${ }^{\mathrm{b}}$ Vitis thunbergii, Vitis thunbergii, Vitis pseudoreticulata, and Vitis hybrid cultivar.

similar to that of the PtWRKY family than the AtWRKY or OsWRKY family, indicating a similar evolutionary history between grape and poplar (Table 3). A phylogenetic tree was constructed based on 58 Group III genes from Arabidopsis, rice, poplar, and grape.

The WRKY genes may act as a regulatory node that plays a crucial role in responses to abiotic stresses or in stressinduced defense signaling pathways [17]. Plant response to pathogens is regulated by multiple signal transduction pathways, in which SA functions as key signaling molecules $[15,38]$. Considerable effort has been directed toward elucidating the regulatory network controlling expression of SA-inducible genes. AtWRKY70 of Arabidopsis thaliana was in the SA-signal transduction pathway leading to PR gene expression [15]. Many of the WRKY genes were responsive to fungal infection and SA treatment, leading us to suspect that they may also play a regulatory role in the establishment of disease tolerance and in the SA signal transduction pathway in grape $[34,39,40]$. Expression analysis of AtWRKY genes in Arabidopsis showed that almost $70 \%$ are differentially regulated in response to pathogen infection and SA treatment, suggesting that the major role of WRKY genes in flowering 
plants is to mediate defense responses [41]. Details of the roles of the WRKY genes in defending dicotyledonous plants against pathogens can be found in three excellent reviews $[4,7,42]$. In our results, WRKY genes in grape showed that almost $57 \%$ (16 genes) were differentially regulated in response to both pathogen infection and SA treatment. It was lower than Arabidopsis. We speculated that the reason was that WRKY genes belong to V. vinifera PN40024 genomic sequence, and the test material was V. vinifera "Pinor," which was susceptible cultivars Europe grape.

\section{Disclosure}

The English in this document has been checked by at least two professional editors, both native speakers of English. For a certificate, please see http://www.textcheck.com/certificate/oPFplV.

\section{Conflict of Interests}

The authors declare that there is no conflict of interests regarding the publication of this paper.

\section{Acknowledgments}

This work was supported by the National Natural Science Foundation of China (no. 31201599) and the Postdoctoral Science Foundation of Henan (no. 93132).

\section{References}

[1] Y. Tao, Z. Xie, W. Chen et al., "Quantitative nature of Arabidopsis responses during compatible and incompatible interactions with the bacterial pathogen Pseudomonas syringae," The Plant Cell, vol. 15, no. 2, pp. 317-330, 2003.

[2] Z. Nimchuk, T. Eulgem, B. F. Holt III, and J. L. Dangl, "Recognition and response in the plant immune system," Annual Review of Genetics, vol. 37, pp. 579-609, 2003.

[3] T. Eulgem and I. E. Somssich, "Networks of WRKY transcription factors in defense signaling," Current Opinion in Plant Biology, vol. 10, no. 4, pp. 366-371, 2007.

[4] B. Ulker and I. E. Somssich, "WRKY transcription factors: from DNA binding towards biological function," Current Opinion in Plant Biology, vol. 7, no. 5, pp. 491-498, 2004.

[5] Y. Zhang and L. Wang, "The WRKY transcription factor superfamily: its origin in eukaryotes and expansion in plants," BMC Evolutionary Biology, vol. 5, article 1, 2005.

[6] P. J. Rushton, J. T. Torres, M. Parniske, P. Wernert, K. Hahlbrock, and I. E. Somssich, "Interaction of elicitor-induced DNAbinding proteins with elicitor response elements in the promoters of parsley PR1 genes," The EMBO Journal, vol. 15, no. 20, pp. 5690-5700, 1996.

[7] T. Eulgem, P. J. Rushton, S. Robatzek, and I. E. Somssich, "The WRKY superfamily of plant transcription factors," Trends in Plant Science, vol. 5, no. 5, pp. 199-206, 2000.

[8] K. Ali, F. Maltese, E. Zyprian, M. Rex, Y. H. Choi, and R. Verpoorte, "NMR metabolic fingerprinting based identification of grapevine metabolites associated with downy mildew resistance," Journal of Agricultural and Food Chemistry, vol. 57, no. 20, pp. 9599-9606, 2009.
[9] P. J. Rushton, I. E. Somssich, P. Ringler, and Q. J. Shen, "WRKY transcription factors," Trends in Plant Science, vol. 15, no. 5, pp. 247-258, 2010.

[10] C. H. Chen and Z. Chen, "Isolation and characterization of two pathogen- and salicylic acid-induced genes encoding WRKY DNA-binding proteins from tobacco," Plant Molecular Biology, vol. 42, no. 2, pp. 387-396, 2000.

[11] L. Du and Z. Chen, "Identification of genes encoding receptorlike protein kinases as possible targets of pathogen- and salicylic acid-induced WRKY DNA-binding proteins in Arabidopsis," The Plant Journal, vol. 24, no. 6, pp. 837-847, 2000.

[12] A. C. Vlot, D. A. Dempsey, and D. F. Klessig, "Salicylic acid, a multifaceted hormone to combat disease," Annual Review of Phytopathology, vol. 47, pp. 177-206, 2009.

[13] W. E. Durrant and X. Dong, "Systemic acquired resistance," Annual Review of Phytopathology, vol. 42, pp. 185-209, 2004.

[14] S. Uknes, B. Mauch-Mani, M. Moyer et al., "Acquired resistance in Arabidopsis," The Plant Cell, vol. 4, no. 6, pp. 645-656, 1992.

[15] J. Li, G. Brader, and E. T. Palva, "The WRKY70 transcription factor: a node of convergence for jasmonate-mediated and salicylate-mediated signals in plant defense," The Plant Cell, vol. 16, no. 2, pp. 319-331, 2004.

[16] D. Yu, C. Chen, and Z. Chen, "Evidence for an important role of WRKY DNA binding proteins in the regulation of NPR1 gene expression," The Plant Cell, vol. 13, no. 7, pp. 1527-1540, 2001.

[17] Z.-J. Guo, Y. Kan, X.-J. Chen, D.-B. Li, and D.-W. Wang, "Characterization of a rice WRKY gene whose expression is induced upon pathogen attack and mechanical wounding," Acta Botanica Sinica, vol. 46, no. 8, pp. 955-964, 2004.

[18] Y. Liu, M. Schiff, and S. P. Kumar, "Involvement of MEK1 MAPKK, NTF6 MAPK, WRKY/MYB transcription factors, COI1 and CTR1 in N-mediated resistance to tobacco mosaic virus," The Plant Journal, vol. 38, no. 5, pp. 800-809, 2004.

[19] R. S. Cormack, T. Eulgem, P. J. Rushton, P. Kochner, K. Hahlbrock, and I. E. Somssich, "Leucine zipper-containing WRKY proteins widen the spectrum of immediate early elicitor-induced WRKY transcription factors in parsley," Biochimica et Biophysica Acta, vol. 1576, no. 1-2, pp. 92-100, 2002.

[20] K. L. Wu, Z. J. Guo, H. H. Wang, and J. Li, "The WRKY family of transcription factors in rice and Arabidopsis and their origins," DNA Research, vol. 12, no. 1, pp. 9-26, 2005.

[21] H. He, Q. Dong, Y. Shao et al., "Genome-wide survey and characterization of the WRKY gene family in Populus trichocarpa," Plant Cell Reports, vol. 31, no. 7, pp. 1199-1217, 2012.

[22] R. B. Ferreira, S. Monteiro, M. A. Picxarra-Pereira, and A. R. Teixeira, "Engineering grapevine for increased resistance to fungal pathogens without compromising wine stability," Trends in Biotechnology, vol. 22, no. 4, pp. 168-173, 2004.

[23] Y. Wang and H. Puchao, "Identification method of resistance of Vitis evaluated to grape anthracnose and grape white rot," Journal of Northwest Sci-Tech University of Agriculture and Forestry, vol. 16, no. 8, pp. 17-21, 1988.

[24] Y. Wang and H. Puchao, "Study of white rot disease resistant mechanism in Chinese Vitis wild species," Northern Horticulture, vol. 1, pp. 1-5, 1988.

[25] M. Jovanovic and M. Sidor, "Some characteristics of the development of Phomopsis viticola epiphytes, carrier of white rot, on the variety Italian Riesling," Savremena Poljoprivreda, vol. 35, no. 9-10, pp. 463-467, 1987. 
[26] O. Jaillon, J.-M. Aury, B. Noel et al., “The grapevine genome sequence suggests ancestral hexaploidization in major angiosperm phyla," Nature, vol. 449, no. 7161, pp. 463-467, 2007.

[27] R. Velasco, A. Zharkikh, M. Troggio et al., "A high quality draft consensus sequence of the genome of a heterozygous grapevine variety," PLoS ONE, vol. 2, no. 12, article e1326, 2007.

[28] J. D. Thompson, T. J. Gibson, F. Plewniak, F. Jeanmougin, and D. G. Higgins, "The CLUSTAL_X windows interface: flexible strategies for multiple sequence alignment aided by quality analysis tools," Nucleic Acids Research, vol. 25, no. 24, pp. 48764882, 1997.

[29] R. Ramamoorthy, S.-Y. Jiang, N. Kumar, P. N. Venkatesh, and S. Ramachandran, "A comprehensive transcriptional profiling of the WRKY gene family in rice under various abiotic and phytohormone treatments," Plant \& Cell Physiology, vol. 49, no. 6, pp. 865-879, 2008.

[30] B. Ulker, M. S. Mukhtar, and I. E. Somssich, "The WRKY70 transcription factor of Arabidopsis influences both the plant senescence and defense signaling pathways," Planta, vol. 226, no. 1, pp. 125-137, 2007.

[31] J. J. Zhang, Y. Wang, and X. P. Wang, "An improved method for rapidly extracting total RNA from Vitis," Journal of Fruit Science, vol. 20, no. 3, pp. 178-181, 2003.

[32] W. H. Landschulz, P. F. Johnson, and S. L. McKnight, "The leucine zipper: a hypothetical structure common to a new class of DNA binding proteins," Science, vol. 240, no. 4860, pp. 17591764, 1988.

[33] Z. Xie, Z.-L. Zhang, X. Zou et al., "Annotations and functional analyses of the rice WRKY gene superfamily reveal positive and negative regulators of abscisic acid signaling in aleurone cells," Plant Physiology, vol. 137, no. 1, pp. 176-189, 2005.

[34] S. Robatzek and I. E. Somssich, "Targets of AtWRKY6 regulation during plant senescence and pathogen defense," Genes \& Development, vol. 16, no. 9, pp. 1139-1149, 2002.

[35] E. M. McInerney, D. W. Rose, S. E. Flynn et al., "Determinants of coactivator LXXLL motif specificity in nuclear receptor transcriptional activation," Genes \& Development, vol. 12, no. 21, pp. 3357-3368, 1998.

[36] S. B. Tiwari, G. Hagen, and T. J. Guilfoyle, "Aux/IAA proteins contain a potent transcriptional repression domain," The Plant Cell, vol. 16, no. 2, pp. 533-543, 2004.

[37] H. Zheng, S. Lin, Q. Zhang, Y. Lei, L. Hou, and Z. Zhang, "Functional identification and regulation of the PtDrl02 gene promoter from triploid white poplar," Plant Cell Reports, vol. 29, no. 5, pp. 449-460, 2010.

[38] J. Glazebrook, "Genes controlling expression of defense responses in Arabidopsis," Current Opinion in Plant Biology, vol. 4, no. 4, pp. 301-308, 2001.

[39] Q. Wang, M. Wang, X. Zhang, B. Hao, S. K. Kaushik, and Y. Pan, "WRKY gene family evolution in Arabidopsis thaliana," Genetica, vol. 139, no. 8, pp. 973-983, 2011.

[40] H. Li, Y. Xu, Y. Xiao et al., "Expression and functional analysis of two genes encoding transcription factors, VPWRKY1 and VpWRKY2, isolated from Chinese wild Vitis pseudoreticulata," Planta, vol. 232, no. 6, pp. 1325-1337, 2010.

[41] J. Dong, C. Chen, and Z. Chen, "Expression profiles of the Arabidopsis WRKY gene superfamily during plant defense response," Plant Molecular Biology, vol. 51, no. 1, pp. 21-37, 2003.

[42] T. Eulgem, "Dissecting the WRKY web of plant defense regulators," PLoS Pathogens, vol. 2, no. 11, article e126, 2006. 

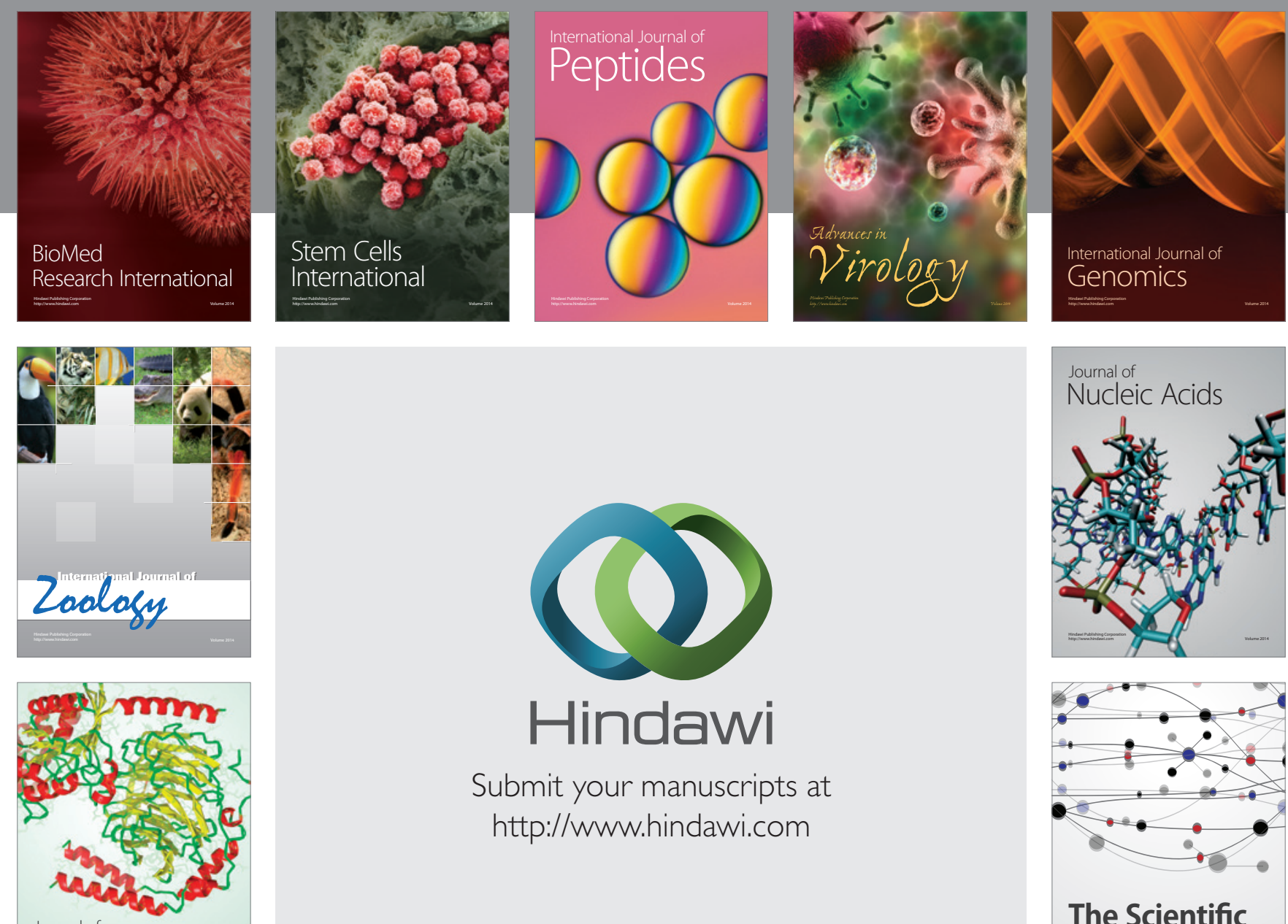

Submit your manuscripts at

http://www.hindawi.com

Journal of
Signal Transduction
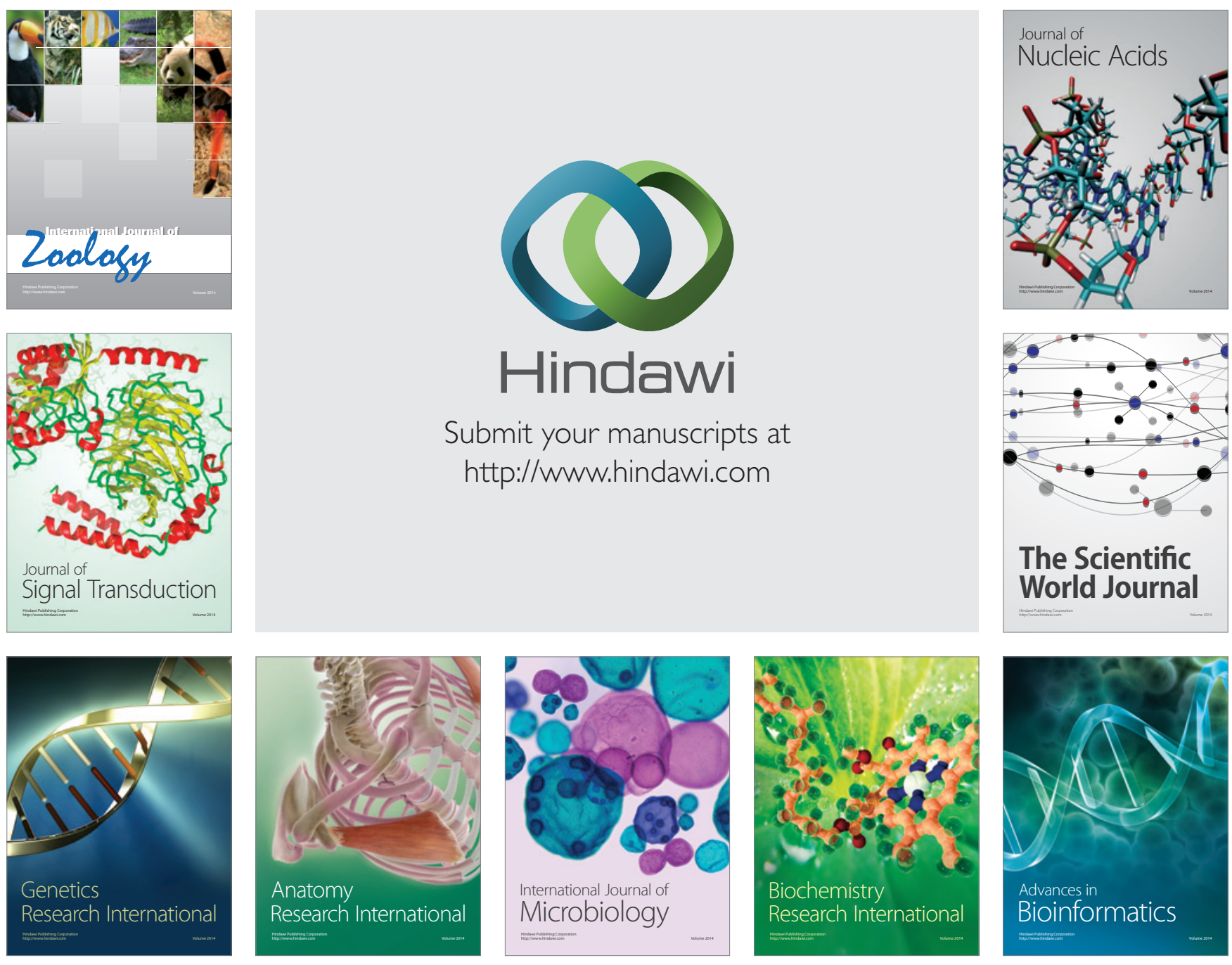

The Scientific World Journal
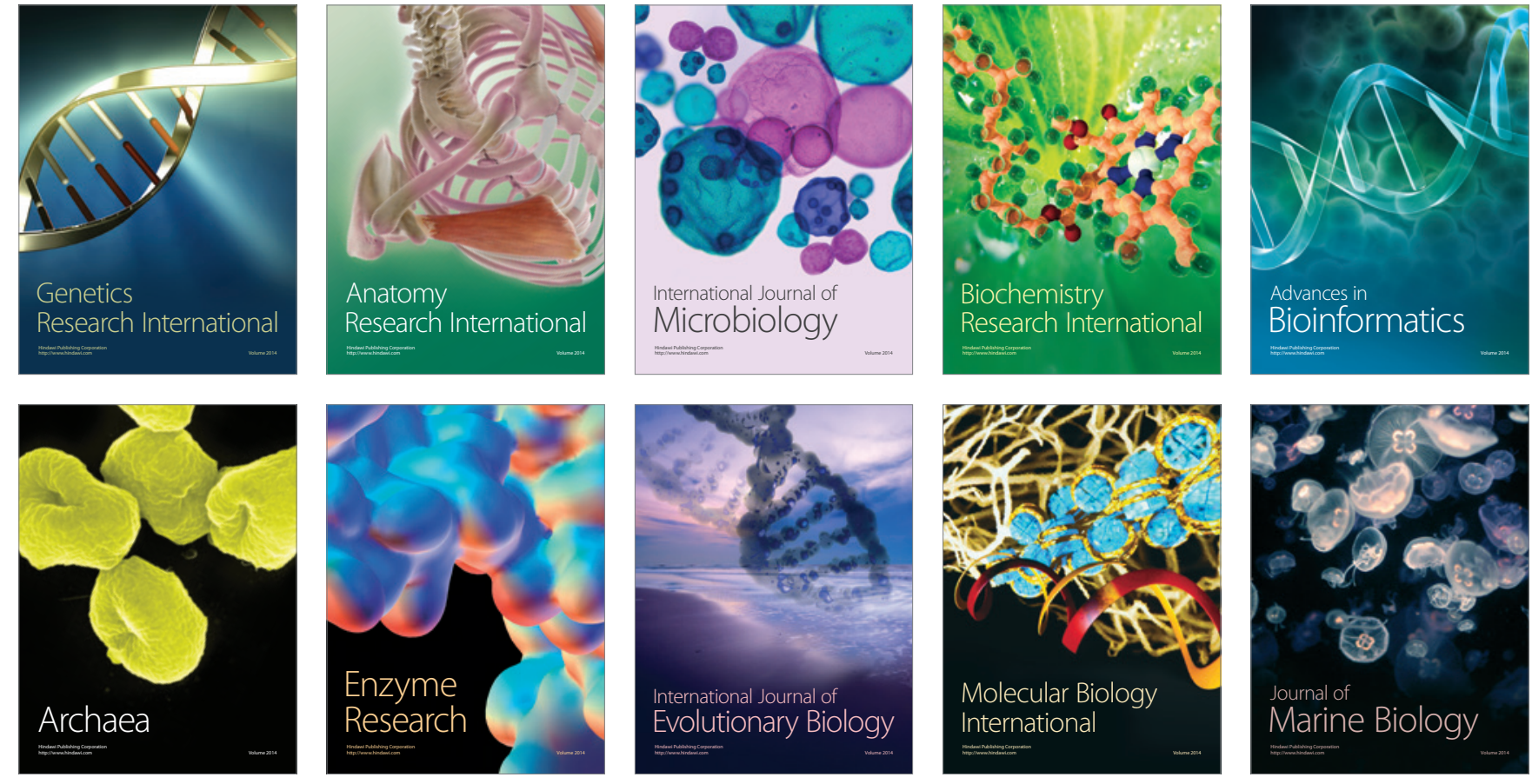